\title{
Additive Manufacturable Materials for Electrochemical Biosensor Electrodes
}

\author{
Moe Elbadawi ${ }^{1}$, Jun J. Ong ${ }^{1}$, Thomas D. Pollard ${ }^{1}$, Simon Gaisford ${ }^{1,2}$, Abdul W. Basit ${ }^{1,2^{*}}$ \\ ${ }^{1}$ Department of Pharmaceutics, UCL School of Pharmacy, University College London, 29-39 \\ Brunswick Square, London WC1N 1AX, UK. \\ ${ }^{2}$ FabRx Ltd., 3 Romney Road, Ashford, Kent, TN24 0RW, UK.
}

*Corresponding author: Basit, A.B (a.basit@ucl.ac.uk).

\begin{abstract}
With the impending Industrial Revolution 4.0, the information produced by sensors will be central in many applications. This includes the Healthcare sector, where affordable healthcare and precision medicine are highly sought-after. Electrochemical sensors have the potential to produce affordable, high sensitivity and specificity, intuitive, and rapid point-of-care diagnostics. Underpinning these achievements are the choice of material and the fabrication thereof. In this review, the different types of materials used in electrochemical biosensors are reported, with a focus on synthetic conductive materials. The review demonstrates that there is an abundance of materials to select from, and compositing different types of materials further widens their applicability in biosensors. In addition, the fabrication of such materials using the state-of-the-art of fabrication technology, Additive Manufacturing (AM), are also detailed. The need for compositing is evident in AM, as the feedstock for certain AM technologies are inherently non-conductive. Both material choice and fabrication technologies limitations are
\end{abstract}


also discussed to highlight opportunities for growth. The review highlights how recent technological advancements have the potential to drive the healthcare industry towards achieving its primary goals.

Keywords: Biosensors; Electrochemical Sensors; 3D Printing; Electrode Design; Point-ofcare.

\section{Introduction}

The role of sensors in driving forward our civilization is immeasurable, where modern civilization has seen an explosion in the ubiquity and necessity of sensors across many aspects of life. Sensors are used in farming, manufacturing, aerospace, computer science, marine and healthcare sectors. ${ }^{[1-6]}$ Sensors can be thought of as artificial extensions of human's natural sensors, which are the visual, olfactory, auditory, touch, and taste sensors. Despite these senses being critical to human survival, they are limited in their detection capacity. For example, in the healthcare industry, sensors have made it feasible to detect pathogens, biological activities and provide feedback on treatments, such as implants, that would otherwise not have been possible without their interjection. ${ }^{[5,7,8]}$ Hence, they provide information that natural sensors cannot. At their core, the information provided by sensors are data, which will be invaluable to the next Industrial Revolution, Industry 4.0. ${ }^{[6,9,10]}$ In Industry 4.0, machine learning and Internet of Things (IoT) will also be important technologies, and both will be reliant on data. A literature search in PubMed® for Electrochemical Sensors reveals that a significant increase in articles has been published in the field since circa 2005 (Figure 1).

As mentioned, the medical field is reliant on sensors, where they are referred to as biosensors. There are different sensing modalities used for biosensors that include physical, light, thermal, acoustic, chemical, and electrochemical. ${ }^{[9,11]}$ The requirements of biosensors are 
similar to that of sensors found in other sectors, which include reliability, cost and sustainability. ${ }^{[12]}$ However, consideration are also given to their biocompatibility, and overwhelmingly so if they incorporated into implants. ${ }^{[13]}$ Of particular interest are electrochemical sensors, which is the fundamental method used by glucose sensors. Diabetic patients, and indirectly healthcare institutes, have tremendously benefited from the technology in terms of both patient quality of life and socio-economical. The first biosensor was developed for oxygen detection in 1956, which was subsequently followed by sensors for glucose and urea in 1962 and 1969, respectively. This eventually led to the first commercial biosensor in 1975. ${ }^{[14]}$ Now, the glucose sensor market was valued at $\$ 12.8$ billion in $2019,{ }^{[15]}$ and is expected to grow due to innovations in portability, cost-effectiveness, and accuracy. Such are the benefits of using electrochemical biosensors. They dominate the glucose market owing to their simplicity, portability, fast response, high sensitivity and low limits of detection. ${ }^{[16]}$ As medicine is moving towards precision medicine and point-of-care diagnostic, the benefits of biosensors will become more evident. ${ }^{[8,17,18]}$ Further research is needed to expand the utility of biosensors to target therapeutic drug monitoring, pathogens and other biological analytes. The ideal sensor for healthcare institutions will need to be fabricated on-demand and needs to be economically viable. Other than healthcare, the food and beverage, water quality control and the sport industry are potential applications for electrochemical biosensors. The significance of the technology being adopted by various and disparate sectors provides the possibilities for new innovations to be translated across; since each sector will have its own approach to problem-solving. Electrochemical sensors as a research field is truly cross-disciplinary and will benefit from being researched by multiple disciplines.

Materials have been fundamental for advancing civilization, and as such several decisive periods were named after materials: Stone, Bronze and Iron Age. More recently, in the Information Age, the role of materials is less apparent. If sensors are crucial to producing 
data and information, then by extension, their fabrication is equally essential. This is also evidenced by the keywords 'carbon', 'graphene' and 'nanoparticles' being among the top 20 keywords in the aforementioned literature search (Figure 1). A network analysis further highlights the intrinsic relationship between both synthetic and natural materials. Therefore, materials are pivotal to the development of electrochemical sensors.

A primary component of EC sensors is the electrode, which interfaces with the analyte of interest. ${ }^{[19]}$ Electrode fabrication is mostly made through conventional techniques, such as screen printing and photolithography, whose limitations are hindering innovation and clinical applications. Conventional approaches are laborious, require cleanroom processing, multi-step processing involving toxic materials, and rely on human involvement and precision. ${ }^{[20,21]}$ Furthermore, conventional techniques are limited to basic designs. Such limitations are not suitable for the high-throughput and precision in fabrication needed for Industry 4.0. More recently, there has been a growing interest in using state-of-the-art in fabrication technology, additive manufacturing (AM), to circumvent these issues. ${ }^{[22-24]}$ This technology offers multiple advantages over traditional techniques, including fabricating complex designs, multimaterial printing, rapid prototyping and high spatial resolution. ${ }^{[25]}$ In addition, AM produces less waste than traditional approaches for manufacturing electronics, making it more sustainable. $^{[26]}$ As a digital fabrication technology, AM can be integrated with other technologies, such as machine learning and machine vision, to automate the fabrication process. ${ }^{[27,28]} \mathrm{AM}$ also enables on-site and on-demand production of bespoke electrodes, which if positioned within a clinical setting, has the potential to meet the demand for point-of-care biosensors. AM is impacting multiple disciplines, where the potential of AM is being realized in both research and clinical/commercial settings. The versatility of AM has resulted in diverse applications thereof in fabricating EC sensors. The primary focus for EC sensors has been on fabricating electrodes, where electrodes with complex structures provide enhanced 
electrochemical performance and reproducibility compared to conventional electrodes. ${ }^{[29,30]}$ In addition, some AM technologies allow for nano-pattern feature construction with greater precision than traditional fabrication technologies, and thus have the potential to address the mutually-exclusive relationship between sintering and loss of nano-features that can impede redox mediating properties when utilizing metallic nanoparticles. ${ }^{[31]}$ As highlighted in this review, AM is capable of fabricating other aspects of EC biosensors, such as electrochemical cells and printed circuit boards.

This review is structured as follows. Section 2 provides an overview of the mechanisms involved in EC biosensors. The main materials used for as electrode transducers are presented in Section 3, with a fundamental explanation of their conductivity. Synthetic materials for enhancing electrode selectivity are presented in Section 4. A detailed progress report of AMbased EC electrodes is presented Section 5, which includes innovative solutions to improving conductivity. The prospect of AM-based sensors is given in Section 6.

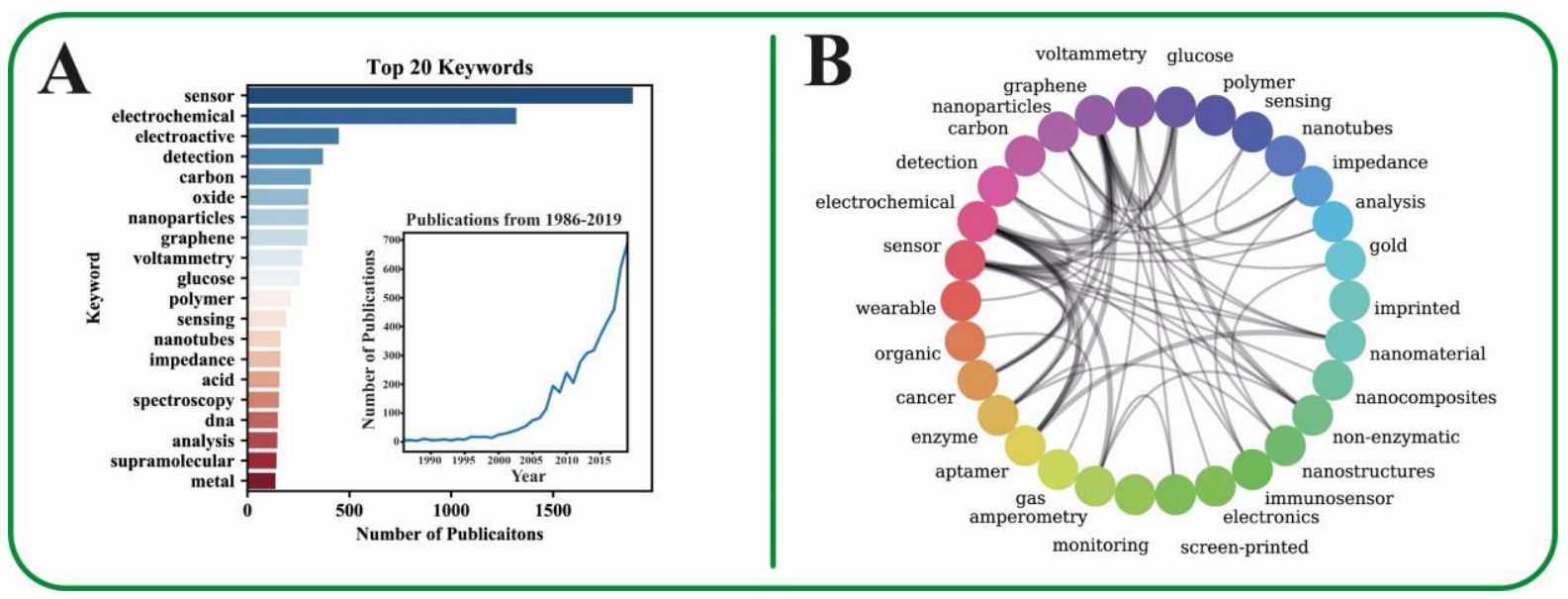

Figure 1. Publications in Numbers. The number of electrochemical sensor publications exponentially increased since circa 2005 (inset in A). The top 20 keywords associated with electrochemistry include carbon, graphene, polymers and nanoparticles, which demonstrates the necessity of materials to electrochemical sensors. The network graph of the keywords reveals the intrinsic link between materials and sensors. Stronger associations are reflected by the width of the edges. (data obtained from Pubmed using 'Electrochemical sensors' as the keyword. Analysis and plotting were developed using the programming language Python). 


\section{Overview of Electrochemical Sensors}

There are a few sensing modalities available when it comes to biosensors. Optical sensors were first used to detect the presence of pathogens. ${ }^{[32]}$ The approach used staining to highlight and differentiate between different cells. This concept of producing a chemical reaction is used to this day, in the form of lateral assay flow (LFA), which is notably used in pregnancy tests. ${ }^{[33]}$ Contemporary applications of light-based techniques have considerably evolved, particularly with the advent of lasers and cameras. Raman in 1928 revealed that lasers could be used to measure the activity of molecules, which led to the discovery of Raman spectroscopy. ${ }^{[34]}$ Collectively, vibrational spectroscopy techniques, which include near-infrared, UV-Visible and FT-IR spectroscopy techniques have been used as modalities for biosensors. ${ }^{[35-37]}$ With the COVID-19 pandemic, thermal imaging techniques were vital in identifying individuals carrying the virus. ${ }^{[38-40]}$ Another modality utilizes thermal energy change. Thermometric sensors measure the energy change associated with a biochemical reaction. The released or absorbed energy is proportional to the number of molecules produced by the biochemical reaction. The temperature change is measured by a microcalorimetry, whereby the transducers are thermocouples or resistance thermometers. The most widely used thermometric sensor are enzymatic biosensors. These have the advantage of simultaneously detecting multiple analytes (multiplex). Thermometric, or calorimetric, sensors have been used to monitor glucose and cholesterol; however, given their high cost, difficulty in manufacturing and possible fouling limit their use. ${ }^{[41]}$ Among the different technologies used thus far, electrochemical-based modalities are the most widely studied, ${ }^{[42]}$ due to their exceptional advantages.

In electrochemistry, the electrical signal is directly generated from the analytical signal, which is in contrast to common spectroscopic methods. Furthermore, optical biosensors suffer from complexity, low sensitivity and ean be difficulty in interpreting the spectra generated. ${ }^{[43]}$ Thus, fewer components are needed within the circuity. Moreover, and in contrast to thermal 
biosensors, electrochemical sensors can be made to detect a specific bioanalyte. ${ }^{[4]}$ The main electrochemical techniques employed in biosensing are amperometry, conductometry and potentiometry. ${ }^{[45]}$ Other techniques include impedance, field-effect transistors, ${ }^{[46]}$ and capacitive sensors have also been employed. ${ }^{[47]}$ Previous work has also sought to combine two electrometric systems into one biosensor. ${ }^{[48]}$

In amperometry, a voltammogram is created to analyse the signal, whereby a potential is generated between electrodes and the change in current is recorded as a function thereof. The change in current is a result of the analyte undergoing either oxidation or reduction. Amperometric detection can be in the form of a two-, three- or four-electrode configuration. The three-electrode setup is more commonly used, comprising a working electrode (WE), a counter electrode (CE) and a reference electrode (RE), as illustrated in Figure 2. The potential in a three-electrode setup is controlled by an electronic circuitry referred to as a potentiostat. The redox reaction causes a change in the current flowing between the WE and CE proportional to the concentration of the bioanalyte. The electrochemical activity occurs on the WE, which results in the oxidation or reduction of the bioanalyte upon receiving a voltage, and where the change in current is measured. Common materials used include platinum, gold, and glassy carbon, with a biorecognition layer incorporated (Section 3). The CE, in turn, behaves as the source or sink of electrons for the cathode or anode WE, respectively, effectively closing the circuit and allowing the current to flow between the two electrodes. The RE is used to accurately measure the applied potential relative to a stable reference reaction. The voltage potential window is a function of the electrode; for example, for a typical carbon electrode, this can be from +1.1 to $-1.2 \mathrm{~V}$, hence the bioanalyte's cathodic and anodic peak potential should fall within the potential window. A number of voltammetry tests can be applied to initiate the electrochemical reaction and generate the electron transfer. Frequently used techniques include 
cyclic, linear, and differential pulse voltammetry. Other electrochemical techniques include conductometry and potentiometry, but are explored to a lesser degree.

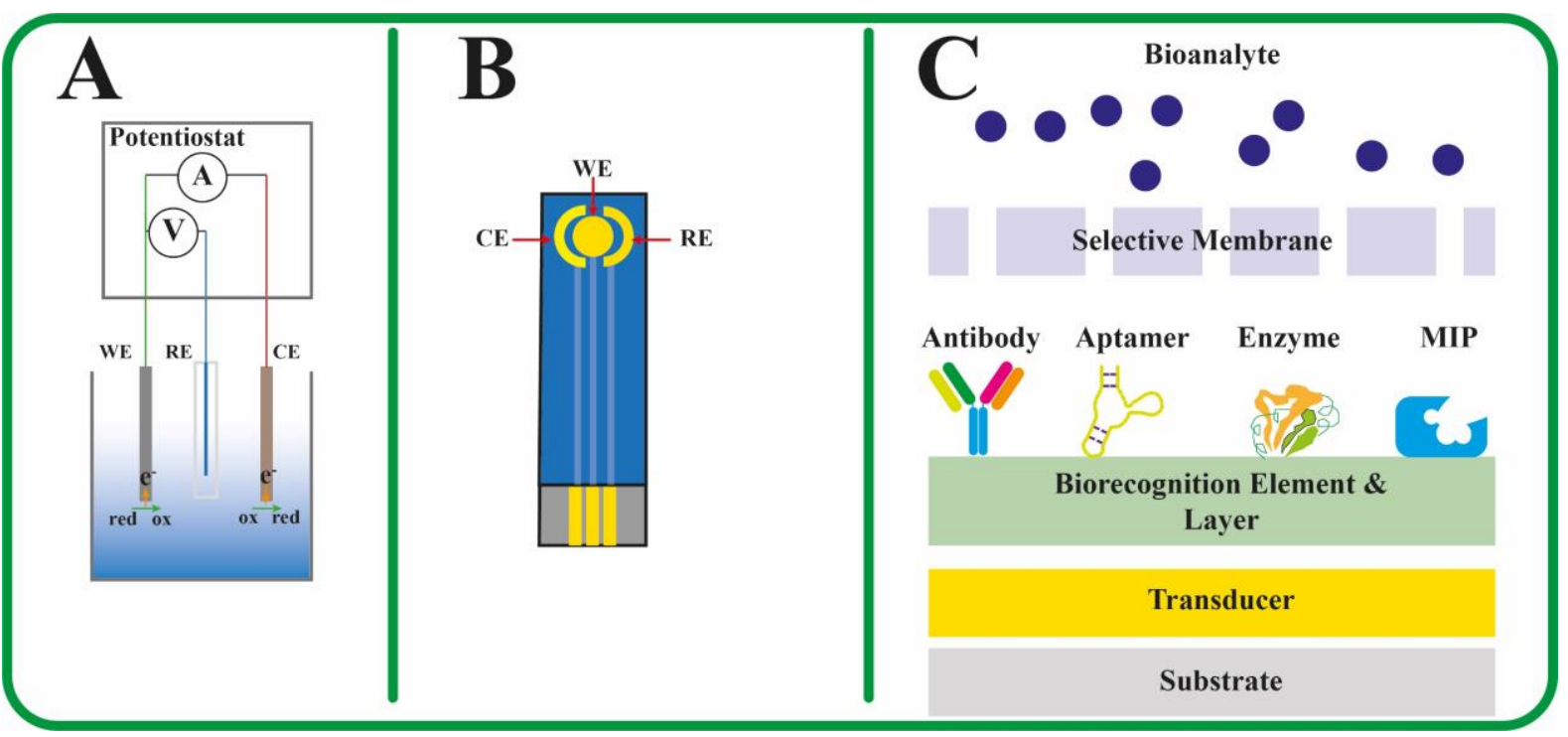

Figure 2 Schematic of (A) an analytical three-electrode potentiostat setup, (B) a screenprinted electrode (WE, CE and RE are the working, counter and reference electrode, respectively), and (C) overview of typical layers of a working electrode (MIP - molecular imprinted polymer).

Most of the research involving electrode design has centered on the working electrode. Conventionally the first layer of the WE is the biorecognition site or layer, if a semi-permeable membrane has not been used. Here, the biorecognition element, which could be natural, namely an antibody, an aptamer or an enzyme; or synthetic, such as nanoparticles or molecularly imprinted polymers, or a combination thereof. ${ }^{[49]}$ In-depth reviews of the biorecognition elements and a selection guide can be found in previous reviews, ${ }^{[49]}$ and a brief discussion of the salient elements are given in Section 0. Suffice it to say, the aim of the element is to form an interaction between the bioanalyte that subsequently generates the electron. Essential characteristics needed include selectivity, whereby only the target analyte generates the electrical signal; sensitivity, with regards to the resolution capabilities of the sensor detecting 
subtle changes; and linearity and detection limit. ${ }^{[50]}$ To achieve said feats, the element needs to be anchored onto the transducer at a high density and in an orientation that allows for the bioanalyte to access the active sites. ${ }^{[51]}$ The layer itself, which is typically polymeric or carbonaceous in form, is used to facilitate the immobilization of the element onto the electrode ${ }^{[51]}$ Key factors to consider include establishing a firm attachment onto the electrode, whilst being durable and non-destructive to the biorecognition element. The material selected will also need to be specific to the desired biorecognition element, and should facilitate the electron transfer. Again, these traits are a function of the material's properties, such as porosity and charge transfer rate, as well as the processing parameters, such as the resultant biorecognition layer height. A thicker layer will be able to house more elements, thereby increasing the density thereof. A commonly-used layer is chitosan, which is biocompatible, capable of forming uniform films and hydrogels, and can be functionalized owing to its multiple oxygen- and nitrogen-based functional group. ${ }^{[52]}$ Furthermore, chitosan is easily sourced and inexpensive.

Adjacent to the biorecognition layer is the transducer. This layer is the conductive component that receives the electrons generated and produces an electrical signal proportional to the biochemical reaction. There are a number of materials used as a transducer, which will be discussed in the following section. From a design perspective, the surface area, surface roughness and porosity of the electrode influences performances. For example, porosity influences immobilization ${ }^{[53]}$ by to imparting a high surface area, ${ }^{[54]}$ and quickens the transport of gas and liquid exchange. ${ }^{[55]}$ The final step is for the electrons to travel across the electrode, and along the traces, and to the potentiostat for readings.

\section{Materials Selection for EC Biosensor Electrode Transducers}

As mentioned, material selection underpins electrode design. Factors governing the material selection include conductivity, cost, availability, workability and biocompatibility. For 
example, an electrode fabricated from gold will achieve a low resistivity, but this comes at the expense of cost. In this section, conventional and emerging materials used to fabricate the transducer are discussed.

\subsection{Metals}

As the most conductive from the main categories of materials, metals are the "gold standard" for many electrical applications. ${ }^{[56]}$ Compared to traditional ceramics and polymers, these values are several orders of magnitude greater. Metals are an inorganic, crystalline materials in which positive ions are surrounded by delocalized electrons, and can form metallic bonds. The metallic bond is a result of the attraction between the positive ions in the lattice and the free electrons. The valence electrons in metals are shared among all atoms rather than associated with an individual atom, and forms what is referred to as an electron gas that randomly circulates the cations. When an electric potential gradient is applied, the electrons move down the gradient with little hindrance, that imparts imparting metals with their high conductivity. Non-crystalline metals can also be synthesized; these and-are referred to as metallic glass (MG), due to their amorphous nature. The advantages of MGs for biomedical application include corrosion resistance, ${ }^{[57]}$ anti-bacterial properties, ${ }^{[58]}$ biocompatibility and biodegradability ${ }^{[59]}$. However, their conductivity is inferior to crystalline metals, ${ }^{[60]}$ and hence have seldom been used as biosensors. It is worth acknowledging that MGs are 3D printable, ${ }^{[61]}$ and that they can be thermoplastically deformed at the nano-level. The latter was exploited by Kinser et al. (2019) using platinum-based BMGs to enhance the signal and sensitivity compared to planar electrodes, for the detection of glucose. ${ }^{[62]}$

For biosensors, metallic nanoparticles (NPs) are the preferred form. ${ }^{[63]}$ Previous work has demonstrated that direct adsorption of enzymes and other proteins on to the bulk metal surface frequently result in denaturation of the protein and loss of catalytic activity. In comparison, metal NPs have a lower denaturing impact. ${ }^{[63]}$ Moreover, they are able to improve 
the conductivity of the electrode owing to their large surface area while insignificantly increasing the occupied volume, in contrast to bulk metals. ${ }^{[64]}$ A further advantage is their ability to provide electrochemical reversibility for redox reactions, which is unattainable with bulk metal electrodes. ${ }^{[65]}$ Additionally, NPs have been incorporated into conductive polymers to enhance the latter's conductivity further. Impedance studies have revealed a considerable decrease in charge transfer resistance on electrode modification. ${ }^{[64]}$ Lastly, 3D printing of metals as NPs is more readily achieved compared to their bulk form. Both gold and silver nanoparticles have been discovered to exhibit conductivities in the same order of magnitude as their bulk counterparts, ${ }^{[66-68]}$ however this is dependent on the processing parameters utilized. Conductivity as high as $31,000 \mathrm{~S} / \mathrm{m}$ was reported when polymeric feedstock was blended with metallic powder for fused deposition modelling (FDM), a subset technology of AM. ${ }^{[69]}$

\subsection{Ceramics}

In electrical circuits, ceramics are widely used as dielectrics where although no or little current is passed through, the electric charges are polarized, and in turn, electrical energy is internally stored. ${ }^{[70]}$ Ceramics can also be doped with either p-type or n-type dopants that can impart conductivity ranging from semi-conductive to conductive. ${ }^{[71,72]}$ The interest in biosensors lies in extrinsic semiconductors, which are materials that are not inherently semiconducting and thereby requiring a dopant. In the case of n-type semiconductors, the ceramic gains loosely bonded electrons through doping. Upon receiving sufficient energy to transition the bandgap, such as in the form of temperature or a voltage, the loosely bound electrons can break its bond from the parent atom and consequently contribute to current flow. ${ }^{[73]}$

An example of an n-type ceramic that has seen widespread use in sensors in the form of thin films is indium-doped tin oxide (ITO), ${ }^{[7]}$ which can also be classified as an alloy depending on the oxygen content. ITO is a composition of indium (III) oxide and tin (V) oxide 
at a ratio by weight of 9:1, wherein the latter is the dopant used to enhance the material's conductivity. The resistivity of ITO can be achieved in the order of $10^{-7} \Omega \bullet \mathrm{m}$, which is one order of magnitude greater than the top three conductive metals. ${ }^{[63]}$ However, the conductivity of ITO depends on the substrate into which ITO is coated on. Owing to its use in solar cells, the primary substrate for ITO coating is transparent quartz glass.; while If flexibility is soughtafter, then ITO is deposited on polyethylene terephthalate (PET), which is also transparent. Aside from its excellent conductivity and low absorbability of light, ITO is less expensive than metallic conductors, exhibits suitable substrate adhesion, and possesses stable electrochemical and physical stability. The low cost of ITO sheets confer disposable electrodes. ${ }^{[75]}$ The primary 3D printing of ITO is in its nanoparticular form via inkjet printing, in which ethanol has been reported to be an effective dispersant. ${ }^{[76]}$ Several studies have obtained resistivity in the order of $10^{-4} \Omega \bullet \mathrm{m}^{[77,78]}$ when inkjet printing, which can be enhanced to $10^{-6} \Omega \bullet \mathrm{m}$ when silver is incorporated, albeit at the expense of transparency. ${ }^{[79]}$ Annealing of inkjet-printed products has been found to govern the final resistivity. ${ }^{[77,80]}$ ITO can also be printed using a particle-free method. The approach entails printing solutions of indium and tin acetate, wherein during the subsequent annealing step carbon dioxide bubbles that are released are able to control the ITO nucleation process. ${ }^{[81]}$ The resistivity determined by the particle-free method was $2.9 \times 10^{-4}$ $\Omega \bullet m$, and yielded a porous film. Aside from ITO, other oxides have been used as electrodes in biosensors. $^{[82,83]}$

\subsection{MAX Phases}

MAX phases are a new class of materials that combine the properties of metal and ceramics. They are materials with the $\mathrm{M}_{\mathrm{n}+1} \mathrm{AX}$. phase, where the $\mathrm{M}$ is an early transition metal; $\mathrm{A}$ is an A-group element, $\mathrm{X}$ is either a carbon and/or nitrogen group, and $\mathrm{n}$ ranges from 1 to $6{ }^{\left[{ }^{[8]}\right.}$ For example, $\mathrm{Ti}_{3} \mathrm{ALC}_{2}, \mathrm{NB}_{4} \mathrm{AlC}_{3}$, or $\mathrm{Cr}_{2} \mathrm{AlC} .^{[84,85]}$ This configuration endows them with unusual 
and exceptional properties, including electrical. The electrical conductivity is due to the metallic bond, which has been measured in the order of $10^{6} \mathrm{~S} / \mathrm{m}$, but can be modified. ${ }^{[86,87]}$ Hadi et al. (2018) applied density functional theory calculations, and reported that conductivity increased as the $\mathrm{M}$ element transitioned down from the top of the periodic table. ${ }^{[88]} \mathrm{A} 2 \mathrm{D}$ derivative of MAX phases, MXene, with remarkable properties can be obtained through exfoliation. ${ }^{\left[{ }^{89]}\right.}$ MXenes were first synthesized in 2011, and have already garnered strong attention due to their high conductivity, which under optimal synthesis condition can yield 1.5 $\mathrm{x} 10^{6} \mathrm{~S} / \mathrm{m} \cdot{ }^{[90]}$ In addition, they have a strong potential for energy storage, with a volumetric capacitance of up to $1500 \mathrm{~F} / \mathrm{cm}^{3} \cdot{ }^{[90]}$ In light of this, their potential in electrochemical sensors has already been investigated, with a selection of examples tabulated in $\operatorname{Re}^{[91]}$. Of note was the use of MXene for inducing direct energy transfer of hemoglobin, which is difficult to obtain on bare electrodes. MXene were also used to enhance the conductivity of PEDOT:PSS, an inherently conductive polymer. ${ }^{[92]}$ Moreover, compared to graphene and its derivatives, the multilayer structure of MXene yields a larger specific surface area, which facilitates immobilization of materials onto the electrode surface. ${ }^{[93]}$ In addition, MXenes were discovered to preserve enzymatic activity on electrode surfaces. ${ }^{[93]}$

MXenes possess the inherent stability properties in liquids, which precludes the need for surfactants or dispersants when forming a stable ink, as would be the case for other particles. ${ }^{[94]}$ Zhang et al. (2019) successfully leveraged this property for both IJP and DIW of titanium carbide MXene inks in both organic and aqueous inks. ${ }^{[95]}$ Incredibly, no sedimentation was observed after 12 months from preparing the ink. With IJP, the authors were able to obtain a conductivity of $2.8 \times 10^{6} \mathrm{~S} / \mathrm{m}$, which decreased to $1.1 \times 10^{6} \mathrm{~S} / \mathrm{m}$ after 1000 bending cycles. The additive-free approach proposed by the authors contributes to the scalability of the conductive material via DIW and IJP. Although currently there is no mention of MXene fabricated using FDM, they have been impregnated into thermoplastic polymers. ${ }^{\left[{ }^{[9]}\right.}$ Thus, there 
is potential for MXene to enhance the conductivity of FDM filaments, provided that the solid loading does not increase the viscosity beyond the printing limits. Interestingly, MXene were revealed to exhibit interesting rheological phenomenon when combined with alginate and poly(ethylene oxide) (PEO). At an MXene solid loading of $1 \mathrm{wt} \%$, the viscosity of PEO/alginate ink decreased by $10.1 \% .{ }^{[96]}$ It was surmised that the inclusion of MXene decreased the interaction between adjacent polymer chains. To the author's knowledge, this is the first report of an inorganic filler having a plasticizing effect; and in contrast to the majority of inorganic fillers (metallic, ceramic, carbonaceous) that increase the viscosity of polymer inks when impregnated therein. Despite being recently discovered, MXenes have a great potential in electrochemical biosensors, with opportunities in wearable technologies. ${ }^{[90,97]}$

A further benefit of MXenes is that they can be bio-functionalized, due to their hydrophilic surface species that allows them to be easily functionalized through either physical adsorption or electrostatic attraction. ${ }^{[98]}$ To date, they have been functionalized with antibodies, DNA, enzymes, and aptamers, to impart features such as enhanced sensitivity or selectivity. ${ }^{[99-}$ ${ }^{103]}$ As Mxenes possess a large surface area, there is potential for immobilization of large amounts of biological molecules.

\subsection{Polymers}

Comparatively, polymers exhibit the highest deformation, and can be easily processed and at lower temperatures and cost. ${ }^{[104,105]}$ A plethora thereof are recyclable as well as biocompatible. For these reasons, polymers are an attractive option for many applications, and have replaced metals and ceramics in certain applications. ${ }^{[106,107]}$ Once again, the conductivity is positively correlated to the carrier mobility and carrier concentration. ${ }^{[108]}$ Indeed, widely used polymers such as polycaprolactone (PCL) and polylactide (PLA) can be made electrically-active by incorporating conductive materials such as carbon nanotubes and graphene once the 
percolation threshold has been reached. However, these will not be discussed hereand. Instead, inherently conductive polymers are the subject of interest for this section.

Inherently-conductive polymers consist of conjugated backbone that give rise to their conductivity, whereby the bonds alternate between single and double carbon bonds. The single bonds have been found to impart strength to the backbone, and the double bonds possess $p$ orbitals that overlap with one another, thereby forming loosely bound electrons that are free to move into a conduction band. Intrinsically, however, such polymers are unstable with respect to bond alternation that induces the formation of a large band gap between the valence and the conducting band (in excess of $1 \mathrm{eV}$ ), which confers semi-conducting characteristics. In order to overcome the large energy gap, and hence transition from semi-conductive to conductive, the conjugated polymers need to doped, whereby a charge carrier is introduced into the polymeric system that either adds or removes electrons. This re-localizes them as polarons or bipolarons, whereupon an electrical potential is applied results in an electric current generated. ${ }^{[109-113]}$ The dopants can be either anionic or cationic, such as $\mathrm{ClO}_{4}^{-}, \mathrm{Na}^{+}$, or larger polymer particles, such as poly(styrene sulfonic acid). ${ }^{[114]}$

There are more than ten conductive polymer systems reported. ${ }^{[115]}$ Poly $(3,4-$ ethylenedioxythiophene) (PEDOT) is one of the more common conductive polymers, and is commercially available. PEDOT possess many advantages that make it attractive for sensors, including biocompatibility, good electrical conductivity (above $300 \mathrm{~S} / \mathrm{m}$ ), prolonged thermal stability at $125{ }^{\circ} \mathrm{C}$, transparent, and stable in air and humidity. ${ }^{[116]}$ PEDOT alone is insoluble, and hence is usually conjugated with poly(styrenesulfonate)(PSS) to bestow solubility ${ }^{[117]}$, and is referred to as PEDOT:PSS. PEDOT:PSS exhibits thermoelectric properties, which can be used for developing energy harvesting technologies. ${ }^{[118]}$ Furthermore, its optical properties provides the added benefit of producing a flexible and transparent electrodes. ${ }^{[119,120]}$ However, pristine PEDOT:PSS exhibits low conductivity (below $1 \mathrm{~S} / \mathrm{cm}$ ) due to the non-conducting PSS- 
rich layers encapsulating the conducting PEDOT chains. ${ }^{[121]}$ However, this can significantly be increased through doping or solution processing. A value of $4380 \mathrm{~S} / \mathrm{cm}$ was reported for PEDOT:PSS when post-treated with sulfuric acid. ${ }^{[122]}$ Dimethyl sulfoxide (DMSO), which incidentally is FDA-approved for intravesical use, ${ }^{[123]}$ has been found to increase the conductivity of PEDOT:PSS through solvent additive methods, with a value of $677 \mathrm{~S} / \mathrm{cm}$ reported. ${ }^{[124]}$ Solvent additive of PEDOT:PSS with ethylene glycol yielded a conductivity of $830 \mathrm{~S} / \mathrm{cm}$, which was ascribed to the increased in carrier mobility from 0.045 to $1.7 \mathrm{~cm}^{2} / \mathrm{VS} .^{[125]}$ A more in-depth review of approaches for enhancing the electrical conductivity of PEDOT:PSS can be found here. ${ }^{[126]}$ Recently, the polymer was solution-processed using a combination of an organic acid-organic solvent which yielded a polymer film thereof with a conductivity of $3500 \mathrm{~S} / \mathrm{cm}$, yet maintaining a high transparency of $94 \% .^{[127]}$ Ionic liquids, which is are of topical interest as solvents in green chemistry, have also been found to enhance the conductivity of PEDOT:PSS, with PEDOT:PSS films possessing conductivity of $\sim 1000 \mathrm{~s} / \mathrm{cm}$ recently discovered. ${ }^{[128]}$ Further processing is also beneficial in addressing PEDOT:PSS hygroscopic behavior, which negatively impacts its conductivity over time, causes the polymer to swell, ${ }^{[129]}$ and has been found to impact the mechanical stability. ${ }^{[130]}$ Furthermore, if impregnated with metallic nanoparticles, then the absorbed water can result in corrosion thereof within the polymeric matrix. ${ }^{[131]}$ The hygroscopic nature of the polymer can be circumvented by forming a neutral-pH PEDOT:PSS that suppressed water absorption, ${ }^{[131]}$ changing the ratio of PEDOT to PSS, ${ }^{[129]}$ and incorporating solid organic compounds. ${ }^{[132]}$ Furthermore, PEDOT:PSS conductivity undergoes degradation upon UV exposure, especially at wavelengths below $320 \mathrm{~nm}$, which can be mitigated via different means. ${ }^{[133]}$ However, this can be leveraged for engineering spatial patterning in film conductivity. ${ }^{[134]}$

Nevertheless, given the aforementioned advantages, PEDOT:PSS has been widely researched in biosensors. ${ }^{[135,136]}$ The polymer displays both excellent electrochemical stability 
and reliability. ${ }^{[137]}$ Additionally, the polymer's surface has been found to be suitable for the electrodeposition of nickel nanoparticles, which is a cheap, selective and sensitive non-enzyme electrode, ${ }^{[138]}$ and glucose oxidase immobilization. ${ }^{[137]}$ Therefore, possessing both attractive conductive and non-electrical properties, the versatility has resulted in PEDOT:PSS in widely being used in biosensors. Other inherently conductive polymers are polyaniline (PANI), ${ }^{[139]}$ polypyrrole (PPy), ${ }^{[140]}$ and polyacetylene, ${ }^{[141]}$ which can be fabricated via AM. For example, PPy AM electrodes have been fabricated for non-amperometric systems, including both wearable and non-wearable supercapacitors. ${ }^{[142-144]}$ Ultimately, AM offers microscale printing of conductive polymers in a programmable and facile manner in $3 \mathrm{D}$ space. ${ }^{[20]}$

\subsection{Carbon Allotropes}

Carbonaceous materials, or carbon allotropes, have been overwhelmingly used in biosensor fabrication, which include graphene (GR), carbon nanotubes (CNT) and carbon black (CB). The sizes of said materials are in the order of nanometers, and compared to other nanomaterials, such as metallic NPs, carbonaceous materials have a larger surface area to volume ratio, high chemical stability, wide potential window, and are chemically inert. Furthermore, they exhibit a rich surface chemistry that provides a variety of redox reactions. ${ }^{[145]}$ The electrochemical characteristics vary between the different allotropes, and are heavily influenced by the hybridization state and the structure thereof. ${ }^{[145]}$

Carbonaceous materials can be classified into zero-, one-, two- or three-dimensional structures. A 2D example is graphene, which possesses exceptional properties that make it ideal as a transducing material. GR has a zero band gap ${ }^{[146]}$ and contains $\mathrm{sp}^{2}$ hybrid bonds in three of the four carbons and that forms overlapping orbitals between adjacent carbon atoms to form a large delocalized pi-bond. ${ }^{[146,147]}$ The effect thereof results in a highly conductive material, ${ }^{[147]}$ which is similar to silver $\sim 10^{8} \mathrm{~S} / \mathrm{m} \cdot{ }^{[148]}$ GR also displays high current density, 
carrier mobility at room temperature, large specific surface area, comparatively high transparency $(97.7 \%)$, large thermal conductivity, and enhanced mechanical properties. ${ }^{[149]}$ One limitation of GR is that it is difficult to synthesize and dispersing disperse in solvents has proven to be challenging. For this reason, previous work has employed derivatives of GR, including graphene oxide (GO) and reduced graphene oxide, that are able to address the aforementioned shortcoming. ${ }^{[145]}$ High-yield synthesis, ${ }^{[147]}$ whilst maintaining fast electron transfer rate and high redox peaks with linear cathodic and anodic currents, ${ }^{[148]}$ can be obtained by said derivative. GO contains a number of oxygen-containing functional groups, which make it highly catalytically active ${ }^{[147]}$. Reduction of GO partially reverts the oxide to its original state and improves its electrical conductivity. ${ }^{[146]}$ Another limitation of graphene is the complex and expensive process to ensure pure GR has been obtained. Catalysts have been utilized to facilitate GR growth however these increase the toxicity after production. ${ }^{[148]}$

Carbon nanotubes are another carbonaceous material that have been intensively explored for electrode fabrication. These are 1D allotropes with a hollow structure and can have one or more walls; an increasing number of walls yields a lower surface area. ${ }^{[150,151]}$ The electrical conductivity of CNTs are again due to the pi-bonds formed between adjacent atoms. CNTs can be simultaneously multi-conjugated to obtain the desired functionality, which has the potential to achieve a multiplex sensor. ${ }^{[152]}$ Conjugation is also necessary to address their limitations, namely insolubility and the tendency to aggregate. Nonetheless, conjugation procedures can be straightforward involving ultrasonication, centrifugation and filtration. Indeed, this depends on whether non-covalent or covalent bonding is desired, whereby the latter is likely to interfere with the conductivity of the CNT system.

The dimensions of CNTs have a diameter that is in the nano-region, which is a requisite, whereas the length can differ, and consequently yield thereby yielding different aspect ratios. Single-walled carbon nanotubes (SWNT) possessing a high aspect ratio with a length of $20 \mathrm{~cm}$ 
has been previously reported. Multi-walled CNT (MWNT) may exhibit diameters of up to 100 $\mathrm{nm}$, where the distance between two walls is comparable to the distance between the graphene layers in graphite. Nevertheless, a typical MWNT has a diameter in the nano-region, and a length in the micron-region. Such small sizes allow CNTs to effectively cross biological barriers, such as crossing the cell membrane and entering individual cells, which is a desirable trait in intracellular biosensing applications. The electronic properties of CNTs are undeniably suited for electrochemical analyses, with an electrical conductivity in the order of $10^{7} \mathrm{~S} / \mathrm{m}$ for pure CNTs. ${ }^{[153]}$ Additionally, and like other allotropes, CNTs display high chemical stability, sensitivity, electrocatalytic activity and fast electron transfer rate. A facet that facilitate conductivity of CNTs include their low defect prevalence, which results in fewer electron scattering. The conductivity of CNTs differ depending on their diameter and chirality, and can either be semi-conducting or semi-metallic. Additionally, CNTs possess large specific surface area that enables immobilization of a number of biofunctional molecules. ${ }^{[154]}$ However, immobilization may damage the activity of a biomolecule, as well as their structural stability. Furthermore, manufacturing the desired CNT dimensions is difficult, in addition to being a costly technique that lacks a high-purity output. ${ }^{[155]}$ Carbon black is another allotrope, albeit not thoroughly as explored as GR and CNTs. CB has a conductivity of $\sim 10^{3} \mathrm{~S} / \mathrm{m}$, which is notably lower than its aforementioned counterparts. However, CB is relatively less expensive, whilst exhibiting stable dispersion without further processing, and can be used to immobilize organic molecules thereto. Again, the conductivity is a function of the $\mathrm{sp}^{2}$ hybridized orbitals, and can be modified through heat treatment. ${ }^{[156]}$

A widely adopted approach in the fabrication of electrodes is to composite polymers, either inherently insulating or conductive, with carbon allotropes to confer the latter's highly conductive attributes. Considerations needed with this approach include the percolation theory 
and the effect of the composite on electron scattering, whereby the latter is inversely proportional to the conductivity. ${ }^{[157-160]}$

It is worth acknowledging the use of a-Another carbon-based material that has seen extensive use in electrochemical analysis, but in the form of a bulk material, is glassy carbon (GC). GC are non-graphitic, vitreous carbon formed as a result of pyrolysis of certain polymeric precursors, ${ }^{[161]}$ that have been employed as analytical electrodes for electrochemical analyses, or as the substrate of the chemically modified electrodes. GC electrodes (GCE) are suitable replacement for metal-based electrodes, such as platinum or gold, as the latter possess a smaller cathodic potential window as a consequence of low hydrogen overvoltage, and thus, are not suitable for redox reactions that occur at more negative potentials. Additionally, the formation of oxides on the surface of platinum and gold electrodes induces high background noises. ${ }^{[162-164]}$ GCE are also favored over graphite electrodes due to the former exhibiting the hardness at high potentials where the latter would physically fail. In addition, GCE exhibit good electrical conductivity with a wide potential window, high hardness, low porosity, low gas and liquid permeability, low oxidation rate, and high chemical inertness. Hence, GCE are suitable as inert electrodes. GCE are, however, susceptible to fouling and adsorbing undesirable matter from the surrounding environment, which include by-products from the electrochemical reaction. GCE possess limited active sites that results in low sensitivity and selectivity of the analyte for trace measurements, hence adsorbing undesirable particulates will further influence the reproducibility of the analysis. For this reason, GCE are seldom used in their bare form and are pre-treated to address their limitations. ${ }^{[165]}$ Pre-treatments are also applied to increase the specific surface area, enhance electron transfer kinetics, and introduce functional groups personalized towards the analyte of interest. Drop cast offers the simplest and fastest method of pre-treating GCE. Using carbonaceous solution establishes $\pi-\pi$ 
interactions with the GCE surface yielding a stable film thereon. ${ }^{[166]}$ Using carbon nanotubes has been reported to increase the sensor lifetime and affords detection at lower potentials. ${ }^{[167]}$

$\mathrm{AM}$ of carbon allotropes such as GR and $\mathrm{CNT}$ is performed through either impregnating or coating a polymer matrix, and via different AM technologies. ${ }^{[168-173]}$ Xiong et al. (2016) demonstrated that AM can be used to spatially control CNT at the nano-level for fabricating nano-architecture electronics. ${ }^{[174]}$ Incorporating carbon allotropes increases the conductivity of polymers, but this is at the expense of increasing the rheological and elastic modulus. Hence, a balance between achieving high conductivity whilst ensuring, for example, extrudability or flexibility is maintained. ${ }^{[69,175-177]}$

\subsection{Quantum Dots}

Quantum dots (QD) is an emerging nanomaterial with 0D, and unique electrical and optical characteristics. $^{[178,179]}$ The majority of the literature indicates that carbon-based QDs are favored for electrochemical biosensors, wherein the carbon atoms are linked by $\mathrm{sp}^{2}$ and $\mathrm{sp}^{3}$ bonds. These amorphous materials display low toxicity and provide a wide functionalizing suitable for surface modifications. ${ }^{[180]}$ In addition, they possess large surface area, excellent biocompatibility, facile and sustainable synthesis, and are relatively inexpensive. ${ }^{[181]}$ In contrast, metal QD are considered toxic. ${ }^{[182]}$ QD have been tested in biological samples for detecting topotecan; ${ }^{[183]}$ the anti-cancer drug cisplatin in both blood and urine samples; ${ }^{[184]}$ and have been impregnated into metal-organic frameworks for improving the sensing activity towards nitrite. ${ }^{[185]}$ A case study of where in which QDs where were incorporated with molecular imprinted polymers is detailed in Section 4.2. QDs exhibit sufficient characteristics that allow them to be fabricated via fused deposition modelling, ${ }^{[186]}$ stereolithography, ${ }^{[187]}$ laser writing, ${ }^{[188]}$ and direct ink writing. ${ }^{[189,190]}$ 


\section{Materials for Enhanced Selectivity}

The ideal electrode should be highly selective for the analyte(s) of interest, in order for the technology to receive practical applications. It is seldom that biological samples reflect the confined restrictions observed in the laboratory; wherein biofluids can possess multiple analytes with overlapping signals. Metallic NP and carbon allotropes can interact with analytes; however, they are less effective when more than one analyte is present. To address this limitation, materials that are highly selective for the analyte of interest are incorporated. Section 2 mentioned examples of such materials, including enzymes, which are used in commercial glucose sensors. This section will focus on two of the most promising synthetic materials, molecular imprinted polymers (MIP), and aptamers.

\subsection{Molecular Imprinted Polymers}

MIPs are polymers with a predetermined 'imprint' of the analyte, which are cavities corresponding to the shape, size and orientation of the bioanalyte. Interestingly, the concept of polymer templates was first proposed in the 1930s. However, it was in the 1970s were when the term MIPs was coined, and that their popularity rose. ${ }^{[191]}$ MIPs are able to achieve high selectivity for a myriad of analytes, including proteins, macromolecular structures and microbes - essentially covering the majority of bioanalytes. ${ }^{[192]}$ Abbasy et al. (2020) recently demonstrated that MIPs can exhibit high selectivity for proteins compared to non-imprinted polymers. ${ }^{[193]}$ Moreover, studies have repeatedly demonstrated their stability across different stresses and biocompatibility, ${ }^{[194-196]}$ whilst demonstrating enhanced retention of analytes. Their potential is further boosted by the low cost and facile preparation methods needed to achieve the high selectivity. Recent work has expanded their application to include molecular recognition in aqueous solutions, which is a positive progress towards their implementation in real-world application. ${ }^{[197]}$ 
MIPs are prepared by first synthesizing the monomer, and subsequently copolymerizing. The monomer is synthesized in the presence of the template (i.e. the desired analyte), which is subsequently solvent washed to leave behind an imprint. The imprint provides both a physical representation of the analyte, and possesses the functional groups needed to establish a bond with future analytes during testing. The polymerization step can be obtained my by different methods, including photo-polymerization, electropolymerization, bulk polymerization and precipitation polymerization. ${ }^{[198]}$

Typically, there are two forms of MIP preparation, which depends on whether a covalent or non-covalent interaction is preferred, with a third, semi-covalent, also reported. The process begins by mixing an admixture of functional monomers, the template (analyte), the initiator and a crosslinker in a solvent. This yields a highly cross-linked polymer that encloses the template, and produces a negative mold thereof. Thereafter, the template is removed from the as-synthesized polymer using a solvent mixture of organic solvent and mild acid. The non-covalent approach is desired, owing to its simplicity in preparation, but also its fast connection kinetics. Regarding the polymerization step, bulk polymerization is the most commonly used approach, however, electropolymerization is also preferred due to the spatial resolution, ease of controlling polymer thickness and reproducibility. ${ }^{[199,200]}$ On the other hand, photopolymerization has a rapid initiation rate, can be performed at low temperature, and offers high spatial polymerization. ${ }^{[201]}$ Spatially controlling the structure of the MIPs can produce patterns with higher surface area, and thereby, result in increased binding sites. ${ }^{[202]}$ This was perfectly demonstrated by Gomez et al. (2016) who fabricated MIPs using two-photon polymerization stereolithography (2PP), which is an AM technology with sub-micron resolution. ${ }^{[203]}$ MIPs were also fabricated using a bioprinter, which operates on a similar principle as direct ink. ${ }^{[204]}$ 


\subsection{Aptamers}

Aptamers are synthetic antibodies that behave like a synthetic receptor for analytes. They are three-dimensional, single-stranded oligonucleotides that synthesized to bind to a specific target. Depending on the configuration, aptamers bind to the target analyte via van der Waals forces, hydrogen bonding, or electrostatic interactions. Their affinity and selectivity are comparable to an antibody, and hence are highly specific. In contrast to antibodies, aptamers can be produced in vitro, and thus obviating the need for animals, easily functionalized, scalable, and do not require low-temperature storage. One notable property of aptamers is their ability to generate easily after denaturation. ${ }^{[205]}$ Their broad application across a number of medical fields is expected to see the aptamer industry reach $\$ 245$ million. ${ }^{[206]}$

The aptamer synthesis process is randomly generated in vitro from a sequence pool, ${ }^{[207]}$ which can take between two days to two weeks to complete. The most common process used is systemic evolution of ligands by exponential enrichment, SELEX, which is an iterative process consisting of incubation, binding, partitioning and amplification. Initially, a random pool of oligonucleotides are is incubated with the desired analyte, wherein some oligonucleotides bind with the analyte. The bound and unbound oligonucleotides are separated during the partitioning step, whereby the bond sequence is further amplified by polymerized chain reaction to enrich the pool. For a more in-depth description of the SELEX process, the following references can be referred to. Refs ${ }^{[208,209]}$

Aptamers can be combined with MIP, where the inclusion of MIP was reported to produce a threefold increase in sensitivity; ${ }^{[210]}$ with a limit of detection in $1 \mathrm{pg} / \mathrm{ml}$ for prostate specific antigen. Recently, a LOD detection for ibuprofen of 33.33 atto-molar was recorded, which is superior to the limit of other analytical techniques such as high-performance liquid chromatography and colorimetry. ${ }^{[211]}$ Hence, there is potential of electrochemical sensors to replace the aforementioned characterization techniques in day-to-day research. It is worth 
noting that aptamers are costlier than MIPs. ${ }^{[212,213]}$ The most frequently used AM technique for aptamers is inkjet printing. ${ }^{[214,215]}$ Aptamers exhibit low thermal stability $\left(<100{ }^{\circ} \mathrm{C}\right),{ }^{[216-218]}$ which precludes their use by high-temperature AM techniques. However, there is potential to improve thermal stability by exploring new aptamer sequences. ${ }^{[219]}$ Their stability during vat polymerization techniques is yet to be determined.

\section{Additive Manufacturing of EC Biosensors}

Electrochemical electrodes have typically been fabricated manually in stages, with screen printing being the common technology used. Screen printing offers rapid production. However, this is at the expense of high production cost, and the electrodes produced being limited to design complexity and two-dimensional. As mentioned in Section 1, AM, also referred to as three-dimensional printing (3D printing), is at the frontier of fabrication; and offers several advantages, including lower production cost, rapid prototyping and can yield electrodes with complex designs.

There are different AM technologies available. The process of AM begins with a model designed using a computer-aided design software (CAD), which-This model is subsequently processed by a slicing software, which allows the AM instrument to compute the layer-bylayer process. The sliced file, in the form of a .gcode, is then sent to the AM instrument where it begins to fabricate the object. Once finished, the object can be removed from the instrument and used as-fabricated. Some technologies however do require a post treatment to produce the final product. Although not necessary, other techniques incorporate post-processing to further enhance the product's properties. The main AM technologies used in EC biosensors are fused deposition modelling (FDM), direct ink writing (DIW), inkjet printing (IJP), aerosol jet

printing (AJP), stereolithography (SLA), digital light processing (DLP), and selective laser 
sintering (SLS) or melting (SLM), where each technique has a different process to fabricating products.

\subsection{Fused Deposition Modelling}

FDM is the most widely used AM technology, owing to its comparatively low capital and running cost, compactness and range of materials available. ${ }^{[220]}$ FDM is an extension of hot-melt extrusion (HME), whereby powdered or pelletized polymers are poured into the hot melt extruder HME. The HME extruder subjects the materials to both heat and shearing before extruding the materials into filaments, which are used as feedstocks for FDM. Filaments can be commercially obtained, including conductive filaments, or can be fabricated in-house for further personalization. When the filaments are inserted into the FDM printer, they are again converted into their semi-molten state and extruded via a nozzle at the designated temperature. As the extrusion process happens, the nozzle, which is on a CNC-type gantry, is moved according to the design made using the CAD software. The semi-molten filament is deposited onto a platform, where it solidifies as it cools down to form the structure. ${ }^{[221,222]}$ FDM offers the possibility to fabricate multi-material objects using dual extrusion, whereby each extruder contains a different material. ${ }^{[223,224]}$ Although the printed product can be used as-fabricated, post-processing of the print can further enhance its properties. Post-processing techniques include physical, chemical and laser treatment. ${ }^{[225-227]}$

FDM uses thermoplastic polymers, which are inherently non-conductive. A conductive print can be achieved through subsequent coating using a conductive paint. ${ }^{[228]}$ However, for the most part, conductivity is achieved by impregnating the filament with a conductive filler,

and subsequently printing. ${ }^{[229,230]}$ A balance must be struck between achieving a conductive filament without comprising its printability. A primary factor is the solid loading of the conductive element. The balance here is to achieve a high solid loading to obtain a percolating 
structure within the polymer matrix, which ensures that current can flow unimpeded. However, the majority of inorganic fillers possess a high elastic modulus, and hence with increasing loading the polymer-filler composite begins to exhibit brittle properties. A previous study demonstrated this by incorporating nanographite into PLA. ${ }^{[231]}$ The nanographite loading ranged from 0 to $40 \%$ (Figure 3 (A)), which was quantified using thermogravimetric analysis. and Scanning Electron Microscopy (SEM), Raman spectroscopy and X-ray photoelectron spectroscopy (XPS) were used to confirm eonfirmed the presence of graphite. During this solid loading range, the PLA-nanographite displayed a sigmoidal increase in conductivity, plateauing at $25 \mathrm{wt} \%$. Incidentally, above this value, the filament became brittle and the printability began to deteriorate due to the lack of thermoplastic binding from the PLA. However, despite being only $25 \mathrm{wt} \%$, the electrode displayed typical graphitic behavior, as demonstrated by the heterogenous electron transfer constant $(k)$ of $8.12 \times 10^{-3} \mathrm{~cm} / \mathrm{s}$. Previous work has also highlighted that increasing the solid loading can result in issues that manifest in different ways, such as nozzle blockage due to high viscosity; accelerated nozzle wear due to the abrasiveness of the materials; and filament fracture due to brittleness. The choice of thermoplastic is also a key determinant. Rymansaib et al. (2016) also used FDM to print their electrodes for detecting lead, ${ }^{[232]}$ and found that at $10 \mathrm{wt} \%$ loading of carbon-nanofibres, polystyrene exhibited higher conductivity than both acrylonitrile butadiene styrene and polycaprolactone, which are two widely-used materials in FDM. Hence, when considering the choice of thermoplastic, considerations should also be given to its wetting, polarity, crystallinity, and melt-viscosity properties, which in turn will influence the electrochemical performance of the FDM-printed electrode. ${ }^{[233]}$ Pre-treating the CNT with a solvent is another alternative to maximizing solid loading, as it can reduce agglomeration within the polymer matrix. ${ }^{[233-235]}$ It is feasible to achieve a solid loading of $53 \pm 7 \mathrm{wt} \%$ for a filament that is both printable whilst functioning as an electrode. ${ }^{[236]}$ 
Further to material properties, the printing parameters are also known to impact electrode performance. For detecting serotonin, Abdalla et al. (2020) reported that the electrochemical activity can be improved by considering the printing orientation (Figure 3(B)). ${ }^{[236]}$ Vertically-printed electrodes exhibited greater anodic current than electrodes printed horizontally. The same observation was also made for outer-sphere redox species, where a larger current peak was obtained through electrodes printed vertically. In addition, the peakto-peak separation was smaller in the vertical orientation. Thus, it was concluded that the vertical orientation should be favored. The same study also investigated the layer thickness for both orientation, where a thickness of $0.1 \mathrm{~mm}$ was superior to $0.4 \mathrm{~mm}$ for both inner and outersphere redox species measurement. The electron transfer rate calculated by the Nicholson method revealed that reducing the layer thickness resulted in significantly faster electron transfer kinetics. Electrochemical impedance studies, which examined the internalized structure of the electrodes, also confirmed that the vertical orientation had the greatest degree of conductivity. The vertical orientation displayed better performance because it was parallel to the electrical connection, and hence, when connecting to a potentiostat, the electrodes should be printed in a parallel orientation to the connecting adapter in order to maximize performance. For layer thickness, a lower value is favored as it minimizes the size of air voids, which form during FDM, but their presence results in increased resistance. Moreover, smaller layer thicknesses can ensure the conductive fillers are more compact, and thus ensuring a complete conductive pathway throughout the print. Previous work has reported a drop of $97.4 \%$ in conductivity between the filament and final 3D printed part (from 31,000 to $820 \mathrm{~S} / \mathrm{m}$ ) ${ }^{\left[{ }^{[6]}\right.}$ Other studies have also reported large decreases in conductivity when the filament was processed via FDM, which was attributed to formation of voids, as well as a lack of sintering between layers. A microporosity of $25-32$ vol\% was sufficient enough to produce the $97.4 \%$ decrease in the 
aforementioned study. Printing parameters such as the nozzle temperature, platform temperature and printing speeds can used to improve layer sintering and minimize voids. ${ }^{[237,238]}$

Evidently, there are multiple parameters to consider, but ultimately FDM is a feasible AM technology for fabricating electrodes. For detecting glucose, ${ }^{[239]}$ Adams et al. (2017) demonstrated comparable performance to industry-standard screen-printed electrodes can be attained. FDM was used to print all three electrodes using a PLA-graphene filament, with a conductivity of $0.6 \Omega \bullet \mathrm{cm}$, onto a mylar substrate, which was subsequently dipped into an enzymatic solution of glucose dehydrogenase flavin adenine dinucleotide (GDH-FAD). The selection of GDH-FAD was a key component to achieving the industry-standard performance, as it is twenty-five times more enzymatic than GOx, and possesses a higher accuracy for glucose detection.

Besides replicating traditional electrode designs, the spatial resolution of FDM can be exploited to produce original electrode designs. Foster et al. (2020) investigated FDMfabricated electrode, where they reported an increase in sensitivity to lead detection with a macroporous electrode compared to a non-porous electrode; ${ }^{[231]}$ due to the former's larger surface area (Figure $3(\mathbf{C})$ ). Commercial FDM printers can be purchased with dual-nozzle functionality, which can be leveraged for a one-step process to print both the insulating and conductive aspects of an electrode. The second nozzle can be used to print a simple casing to insulate the conductive tracks, ${ }^{[232]}$ but there has been recent interest in developing hybrid electrochemical fluidic biosensors using FDM. These biosensors allow for more control over fluid dynamics, which yields a number of advantages, including enhanced accuracy and selectivity, portability, and real-time and high-throughput detection. ${ }^{[240]}$ Katseli et al. (2019) used FDM to develop an electrochemical cell for the simultaneous detection of both paracetamol and caffeine (Figure $3(\mathbf{D})$ ). The limit of detection were 0.43 and $0.39 \mathrm{mg} / \mathrm{L}$ for paracetamol and caffeine, respectively, which were in the range obtained by other 
electrochemical sensors fabricated through traditional means. ${ }^{[241]}$ The team estimated the cost of one electrochemical cell to be $\$ 0.11$, which demonstrates that FDM is an economically viable approach to fabricating electrochemical cells. More recently, FDM was used to fabricate electrochemical cells that coupled voltammetry with Raman spectroscopy, where both sensing techniques offer complimentary information regarding the analyte. ${ }^{[242]}$

Post-processing of additively manufactured electrodes has been widely adopted as a strategy to enhance conductivity. Post-processing of FDM parts has been applied for other applications, but here it can circumvent the issue of low solid loading of conductive fillers, whilst leveraging the advantages of FDM. It can take the form of either subtractive or additive post-processing. The former usually entails the removal of the non-conductive polymer matrix to expose more of the conductive filler to the bioanalyte. The process can be achieved through either mechanical polishing, thermal termination, electrochemical activation or laser ablation. Electrochemical activation has been regularly employed as a subtractive post-processing technique. Rocha et al. (2020) fabricated a Ni-G-PLA electrode, and found that a 36-fold increase in anodic peak can be achieved using a combination of polymer saponification and electrochemical activation in the presence of $0.5 \mathrm{~mol} / \mathrm{L}$ sodium hydroxide. ${ }^{[243]}$ This was verified using SEM micrographs that revealed the process partially removed the PLA, whilst exposing Ni particles. In addition, electrochemical activation can be used with carbonaceouscomposited filaments to form reduced graphene oxide. ${ }^{[244]}$ Marzo et al. (2020) studied the effect of the activation time on PLA-G FDM electrodes, where the conductivity was found to increase with increasing activation time, with a notable increase in the first 150 seconds. ${ }^{[245]}$ The formation of reduced graphene oxide functionalized with $\mathrm{O}-\mathrm{C}=\mathrm{O}, \mathrm{C}=\mathrm{O}$ and $\mathrm{C}-\mathrm{O}$ was confirmed by X-ray photoelectron spectroscopy (XPS). Hence, electrochemical activation has a bimodal effect to enhancing conductivity, by degrading the non-conductive polymer and functionalizing conductive fillers. 
Further to activation time, the activation potential and solvent are known to affect the final conductivity. ${ }^{[246]}$ Santos et al. (2018) thoroughly investigated the physicochemical effects of electrochemical activation on PLA-graphene FDM electrodes. ${ }^{[247]}$ The study investigated activation using a single anodic activation voltage $(1.8 \mathrm{~V})$ and a range of cathodic potential values $(0$ to $-1.8 \mathrm{~V})$. SEM imaging highlighted once again that a rough surface had formed following activation due to polymer degradation, irrespective of activation voltage used (Figure 3 (E)). Atomic force microscopy (AFM) confirmed that the overall roughness did indeed increase at the microscale, but also illustrated homogenous and smooth surfaces at the nanoscale compared to the as-printed electrode. Kelven probe force microscopy (KPFM) imaging revealed an increase in surface electric potential from 0 to $0.7 \mathrm{~V}$ following activation, with a more uniform electric distribution observed when using -1.8 V. To get a better understanding of the change in surface electric potential, Raman spectroscopy was conducted. Observing the D bands, indicative of structural defects, and G bands, it was observed that -1.8 $V$ had a ratio $I_{D} / I_{G}$ of 0.825 , which was notably higher than both the as-printed and anodictreated electrode. Hence, a cathodic potential of $-1.8 \mathrm{~V}$ resulted in an increase in the number of defects, which was caused by increase fragmentation of the graphene sheets and presence of large amounts of small size $\mathrm{sp}^{2}$ domains. For measuring dopamine, an increase in the number of $\mathrm{sp}^{2}$ domains facilitated the interaction between the electrode and the bioanalyte via $\pi-\pi$ stacking. It is worth remarking that the $\mathrm{I}_{\mathrm{D}} / \mathrm{I}_{\mathrm{G}}$ peak intensities did not show a linear increase with increasing cathodic potential, which suggests that other defects occurring on other atomic domains, such as $\mathrm{sp}^{3}$.

Ultimately, electrochemical activation results in a clean electrode surface, and the process is reproducible, stable, and inexpensive. ${ }^{[24,248]}$ Immersing the electrode in solvents has also been reported to improve the sensitivity of AM electrodes, albeit the process required 10 minutes, which is more than double that of electrochemical activation. ${ }^{[249]}$ SEM micrographs 
again confirmed that the conductive filler was superficially exposed following solvent immersion. It is worth noting that the use of electrochemical activation is effective only where the electrochemical analysis itself does not include an electrochemical pre-treatment step, such as square wave voltammetry. ${ }^{[231]}$

In contrast, additive post-processing involves the formation of conductive elements onto the surface of the electrode. Such techniques include physical vapor deposition and chemical deposition, but the most commonly used approach is electroplating. The rationale behind electroplating is that the technique is straight word to implement, controllable, rapid and cost-effective. The most significant finding was that by Vaneckova et al. (2020), who reported conductivity comparable to both conventional metallic- and carbon-based electrodes when electroplating copper onto PLA-Cu FDM prints. ${ }^{[250]}$ This is a noteworthy discovery as it demonstrates the feasibility of AM-based electrodes: conductivity similar to traditional electrodes can be attained, whilst retaining the advantages of AM (complex shape, rapid prototyping, etc.). A low voltage of $-6 \mathrm{~V}$ was used for electroplating, which was found to form a non-porous structure, which is detrimental to achieving high conductivity. SEM imaging revealed good coverage of $\mathrm{Cu}$ particles onto the electrode surface, and hence the purer $\mathrm{Cu}$ surface enhanced both intrinsic kinetic barrier for the electron transfer, and increased total active site surface area. The group repeated the same strategy for polylactide-carbon black FDM prints, and reported faradaic peak separation values superior to that reported by any FDM printed polylactide-based electrode thus far. ${ }^{[251]}$ A separate group investigated the electroplating of both $\mathrm{Cu}$ and Ni onto FDM electrodes, for the detection of glucose. ${ }^{[252]}$ SEM micrographs revealed electroplating to form good coverage of both $\mathrm{Cu}$ and $\mathrm{Ni}$ on the electrode's surface, with average particle size of 2.31 and $1.4 \mu \mathrm{m}$, respectively. Energydispersive $\mathrm{X}$-ray spectroscopy (EDX) detected the presence of either $\mathrm{Cu} / \mathrm{Ni}$, with both oxygen and carbon peaks. A follow-up with XPS revealed that the $\mathrm{Cu}$ likely to have reacted with the 
oxygen to form copper oxide. The study did not mention whether the oxidation occurred during the fabrication process, but if so it should be mitigated where possible as oxidation of $\mathrm{Cu}$ is known to negatively impact its conductivity. ${ }^{[253]}$ Furthermore, hydroxides of both $\mathrm{Cu}$ and $\mathrm{Ni}$ were also detected using XPS, which is likely to be a byproduct of electroplating. For example $\mathrm{Cu}(\mathrm{OH})_{2}$ is known to form in the presence of copper sulfate, ${ }^{[254]}$ which was used as the electrolyte for the $\mathrm{Cu}$-plated electrode. The presence of nickel hydroxide was desirable for this study, as it was postulated by the authors that the oxygen atom from the hydroxide was able to interact with the hydroxyl group of glucose, and thus promoted the dehydrogenation of glucose. However, The Ni-plated electrode resulted in a narrow oxidation window, which was unsuitable for the detection of glucose using cyclic voltammetry. The authors addressed this issue by using the more sensitive technique of chronoamperometry to sense glucose. Nonetheless, the finding of this study suggest that more work is needed to investigate the parameters of electroplating to achieve optimal conductivity.

Other than metallic electroplating, electropolymerization can be applied where a conductive polymer film is formed. In order for either electroplating or polymerization to work, the 3D printed part will need to have a conductive surface. For a non-conductive printed part, then other additive platforms, such as supersonic cluster beam deposition (SCBD) can be used. Gebreyes et al. (2020) demonstrated that it was feasible to combine FDM with SCBD to develop an electrochemical cell. ${ }^{[240]}$ There is also the possibility to explore other postprocessing approaches that are known to modify the surface area, and offer controlled pyrolysis for carbonization of surfaces. ${ }^{[226,227]}$

Overall, FDM is a viable AM technology for researching electrodes. The technology offers fast fabrication, tailorable designs and exhibits electrochemical properties comparable to that fabricated using traditional techniques. Moreover, hybrid structures in a one-step manner can be fabricated, which further expands their application. Within a research context, 
all these advantages are welcomed. Several strategies are being investigated, but the most promising is the use of post-processing to enhance conductivity. Future work is needed to explore the feasibility of incorporating a biorecognition layer, and how this layer would be affected by different processing strategies. Recent work has shown that low temperature FDM printing $\left(<100{ }^{\circ} \mathrm{C}\right)$ can be obtained, which will open the opportunity to incorporate aptamers, and thereby improve electrode selectivity. ${ }^{[255,256]}$ Improving the selectivity should be the focus of near-future research using this AM technology. [257]

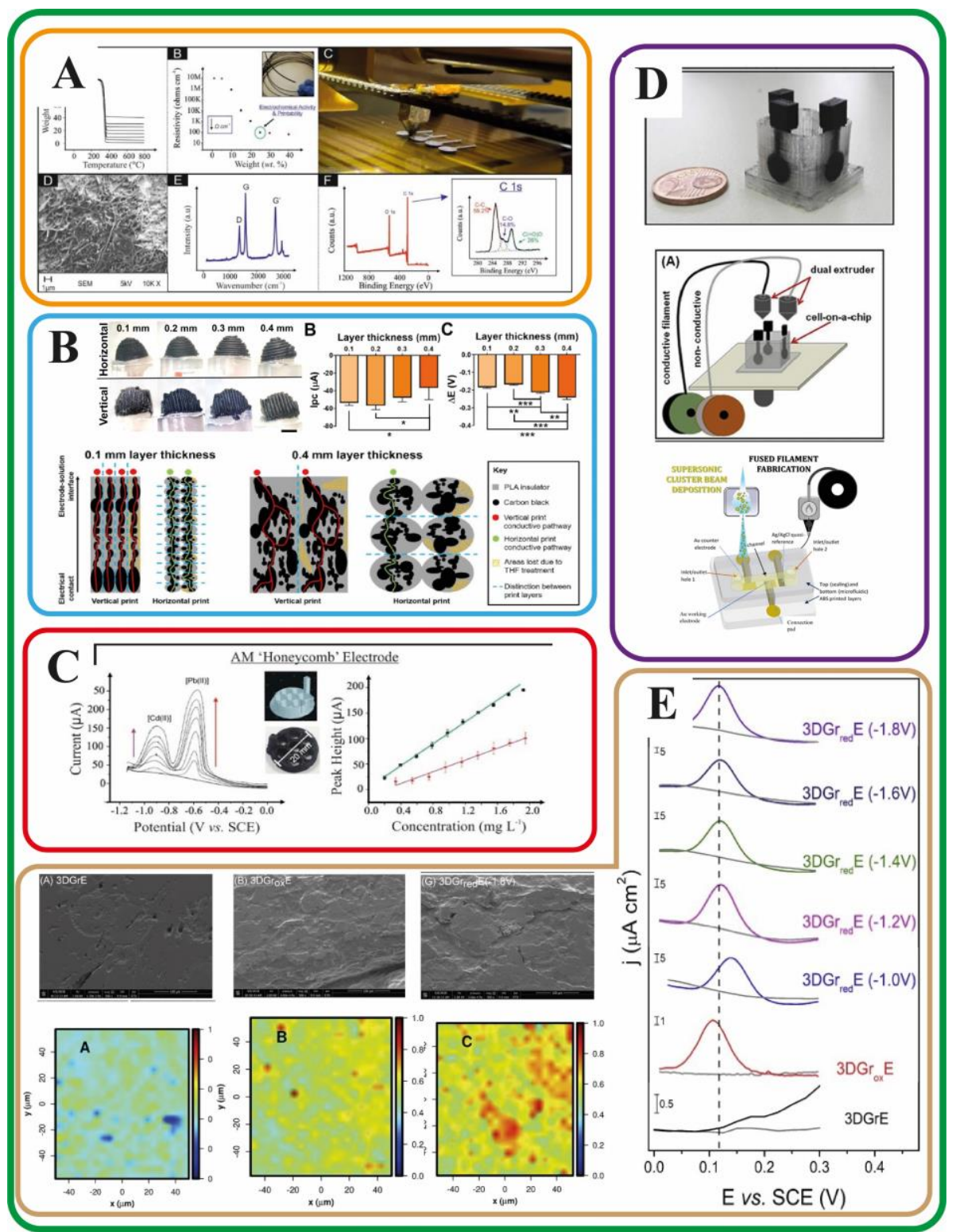

Figure 3. FDM-based electrodes. Parameters such as filler solid loading are critical to achieving a working filament (A). ${ }^{[231]}$ Subsequently, the printing orientation also influences electrochemical performance (B). ${ }^{[236]}$ FDM can fabricate novel electrode design to improve 
performance $(\mathbf{C})^{[231]}$ and can also print electrochemical cells (D). ${ }^{[240,241]}$ If needed, postprocessing is incorporated to modify the surface of the electrode to enhance electrochemical performance $(\mathbf{E}) .{ }^{[247]}$ All figures were adapted and reproduced with permission from Elsevier,

B.V.

\subsection{Direct Writing Extrusion Techniques}

Direct writing techniques are extrusion-based AM technologies that include direct ink writing (DIW), inkjet printing (IJP) and aerosol jet printing (AJP). They are also widely researched, with their primary strength over other AM being their versatility to accommodate a wider range of materials. ${ }^{[258,259]}$ For example, DIW has been used to fabricate polymeric, ceramic and metallic scaffolds. ${ }^{[260]}$ Direct writing technologies deposit inks onto a platform, which are either mechanically-, pneumatically- or thermally-actuated. These technologies do not necessarily demand high temperatures for actuation, and hence are capable of accommodating biological elements, including materials used for biorecognition layers. Another advantage with these technologies is that a higher solid loading can be achieved in comparison to FDM, since the viscosity of the polymer matrix is considerably lower. Hence a higher conductive filler loading can be achieved to obtain higher conductivity.

\subsubsection{Direct Ink Writing}

Compared to traditional fabrication techniques, DIW, also referred to as robocasting, is faster, cheaper and more customizable. ${ }^{[261]}$ DIW, in contrast to FDM, is capable of printing inherently conductive polymers without needing a secondary polymer. In addition, it has a higher resolution than FDM, and can print electrodes with traces with a width of approx. $30 \mu \mathrm{m}$, making DIW suitable for printing fine features. ${ }^{[262]}$ For example, electrodes small enough for measuring glutamate concentration on mouse brain slices have been printed (Figure 4 (A)). For producing fine features, the operating viscosity range is narrower than FDM, and generally limited to nano-sized particles. The trace width is influenced by both the speed of writing and pressure generated. $^{[262]}$ 
Yuk et al. (2020) recently demonstrated that a facile electrode can be fabricated, with a comparatively high degree of flexibility and spatial resolution. ${ }^{[20]}$ The ink used was comprised of PEDOT:PSS nanofibrils dissolved in dimethyl sulfoxide (DMSO), where the researchers were able to establish an operating rheological window. Below this window the traces were found to spread and lose their structural fidelity, which was counteracted by increasing the concentration of the nanofibrils. However, the concentration of PEDOT:PSS was limited to 7 wt $\%$, as any higher resulted in nozzle clogging. Small angle X-ray scattering revealed that the distance between the PEDO-rich crystalline domain decreased with increasing PEDOT:PSS nanofibrils, which led to an increase in interaction between adjacent nanofibrils. With this operating window, a remarkably low resistivity of $6.5 \times 10^{-3} \Omega \bullet \mathrm{cm}$ was recorded, which was obtained by using smaller nozzle diameter. It was reported that the increased shearing effect improved the nanofibril alignment, which is an advantageous inherent property of extrusionbased AM techniques as alignment of fibres, or fillers with a high aspect ratio, can improve connectivity. The study further demonstrated the practicality of DIW for high-throughput fabrication by printing over 100 circuit patterns onto polyethylene terephthalate substrate in under 30 minutes, and with a high degree of reproducibility.

Dong et al. (2018) used DIW to fabricate a novel electrode design for the detection of lactate. ${ }^{[263]}$ The conductive material used therein was AgNPs, with a resistivity in the order of $10^{-6} \Omega \bullet \mathrm{cm}$. The ink printed onto a PET substrate, which was subsequently annealed at $100{ }^{\circ} \mathrm{C}$ to improve the conductivity thereof. The WE was drop-casted with Nafion to exclude interfering anionic species, such as ascorbate, from affecting the signal. This was achieved due to Nafion's negatively charged groups. Once the Nafion layer was allowed to dry, lactate oxidase was deposited, which was immobilized by bovine serum albumin. The RE was fabricated by chloridizing the AgNP-printed electrode in Clorox bleach for one minute, whereas the CE was an unmodified Ag-NP-printed electrode. Hence, compared to the work 
performed by Adams et al., a more conductive electrode was fabricated with care given to the stability of the RE.

DIW versatility is further highlighted by the possibility that it can print onto any surface, including both planar and non-planar surfaces. ${ }^{[264]}$ Of particular interest are flexible surfaces, which are gaining interest for wearable technologies. DIW-printed electrodes were found to preserve their conductivity following 10,000 cycles of repeated bending tests (Figure $4(\mathbf{C})) \cdot{ }^{[20]}$ In fact, recent work reported that bending the DIW-printed electrodes can increase the sensitivity for lactate, glutamate and glucose by under 10\% (Figure 4 (B)). More research is needed to see if greater increases in sensitivity can be achieved, which could consequently lead to a new strategy of increasing sensitivity without, for example, needing to increase filler content. $^{[261]}$ Regarding, post-processing of DIW-printed electrodes generally entails solvent evaporation, but for improved electrochemical performance, electrochemical activation can also be used. ${ }^{[265]}$ 


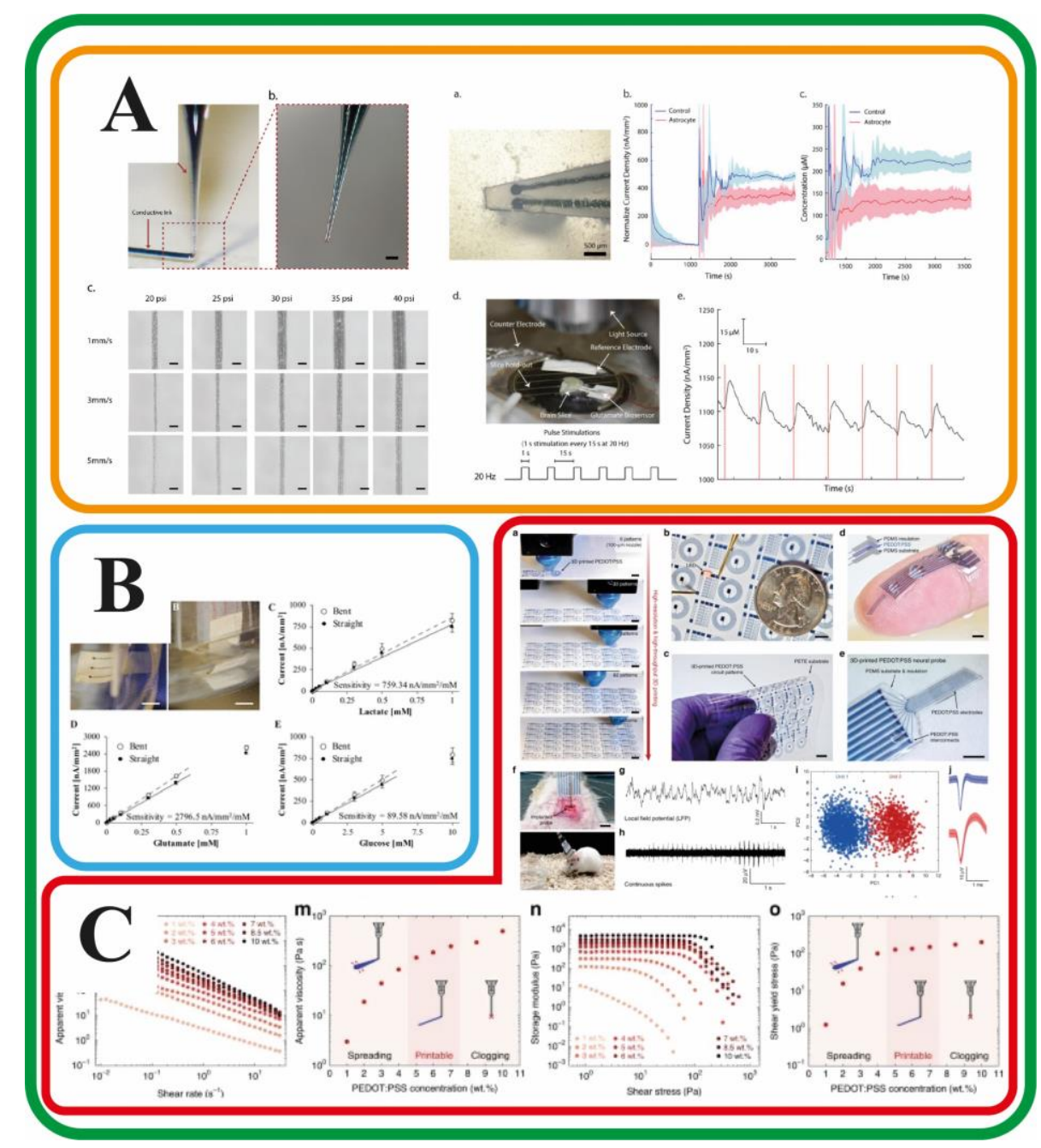

Figure 4. DIW-based electrodes. (A) illustrates the fine traces produced, which are suitable for measuring signals in mouse brain slices. ${ }^{[262]}(\mathbf{B})$ is the effect of bending on electrode current. $^{[261]}(\mathbf{C})$ highlights the need to determine the optimal rheological window to achieve electrodes with suitable traces. ${ }^{[20]}$ Figures were adapted and printed with permission from Elsevier B.V., and Springer Nature.

\subsubsection{Inkjet Printing}

Inkjet printing has also been widely used in fabricating electrodes for amperometric systems. Electrodes fabricated using IJP have been demonstrated to produce better stability and reproducibility when compared with conventional electrodes, in addition to being a rapid, userfriendly and inexpensive fabrication technology. ${ }^{[30]}$ Electrodes were also found to possess greater sensitivity than that fabricated via thin-film deposition for detecting hydrogen peroxide, due to the greater surface roughness of the electrode fabricated by IJP. ${ }^{[266]}$ 
IJP operates with viscosities in the order of $<100 \mathrm{mPa} . \mathrm{s}$, which is notably smaller than other extrusion-based AM technologies. ${ }^{[267]}$ For example, the ideal viscosity range for FDM is in the order of $10^{2}$ Pa.s, which is magnitudes greater than IJP. ${ }^{[268,269]}$ Further to viscosity, there is also an operating window for ink surface tension, where below a given value the formulation will prematurely leak, whereas above the operating window the droplets are expelled from the cartridge. The particle size is another key determinant of printability, where it is advised to use particles in the order of nanometers to prevent nozzle clogging. Typically, multiple layers are deposited per one trace to improve conductivity and thus current response, whilst maintaining thicknesses in the order of nanometers. ${ }^{[270,271]}$ The effect of IJP layers on electrochemical performance was recently investigated using graphene ink. ${ }^{[272]}$ An electrochemical signal was detected using two deposited layers, however, subsequent increases in layers was discovered to increase both the peak and capacitive currents. This inferred that the real surface area and overall porosity increased as more layers were deposited. As a function of layers, the current increased linearly, until the tenth layer where thereafter the signal began to plateau. Ten layers was selected for electrode fabrication as it exhibited the smallest peak-to-peak separation.

Other than graphene, IJP is used to fabricate electrodes made using either metallic nanoparticles, which will subsequently need to be sintered to achieve a conductive path; as well as conductive polymers. In addition, inherently conductive polymers can also be used. Weng et al (2014) reported the inkjet printing of PPy-based biosensor for the detection of glucose. ${ }^{[273]}$ The oxidizing agents, horseradish and glucose oxidase, were dispersed within the PPy solution. Additionally, inkjet printing was used to print a permselective membrane comprised of ethyl cellulose. The glucose detection range was found to be 1-5 mM.

Another appeal of IJP is that a one-step electrode can be employed to fabricate the different layers depicted in Figure 5 (A) and (B), which can expedite the fabrication process. As an example, Bihar et al. (2018) fabricated an all inkjet-printed disposable glucose sensor, 
using paper as the substrate. ${ }^{[274]}$ This encompassed the electrical components, including the contact pads, using PEDOT:PSS with a conductivity of $250 \mathrm{~S} / \mathrm{cm}$; a UV-curable dielectric ink as an insulating layer; a biorecognition layer comprised of GOx, ferrocene complex to facilitate electron transfer; and a selective membrane using nafion. The ability to print selective membranes such as nafion improves the selectivity of electrode. In this study that focused on glucose, uric acid and glucose shared a similar anodic peak during cyclic voltammetry, where nafion was able to reduce the peak by $84 \%$. The study concluded by demonstrating it was feasible to detect glucose in saliva within physiological values of interest.

Beyond replicating SPE-like electrodes, recent progress has gone into producing flexible sensors using IJP, with an interest toward wearable sensors. ${ }^{[275]}$ Typically, the inks are deposited on flexible substrates such as PET or paper, where depending on the substrate the inks will exhibit different adhesion. Prior work revealed that different ink formulations used to increase conductivity, primarily through preventing particle agglomeration, will decrease adhesion strength. ${ }^{[276]}$ When factoring that the conductivity is negligibly affected following long-term bending tests, IJP presents an attractive AM technology for producing wearable sensors. Examples of flexible electrodes are presented in Figure $5(\mathbf{C})$ and (D). 


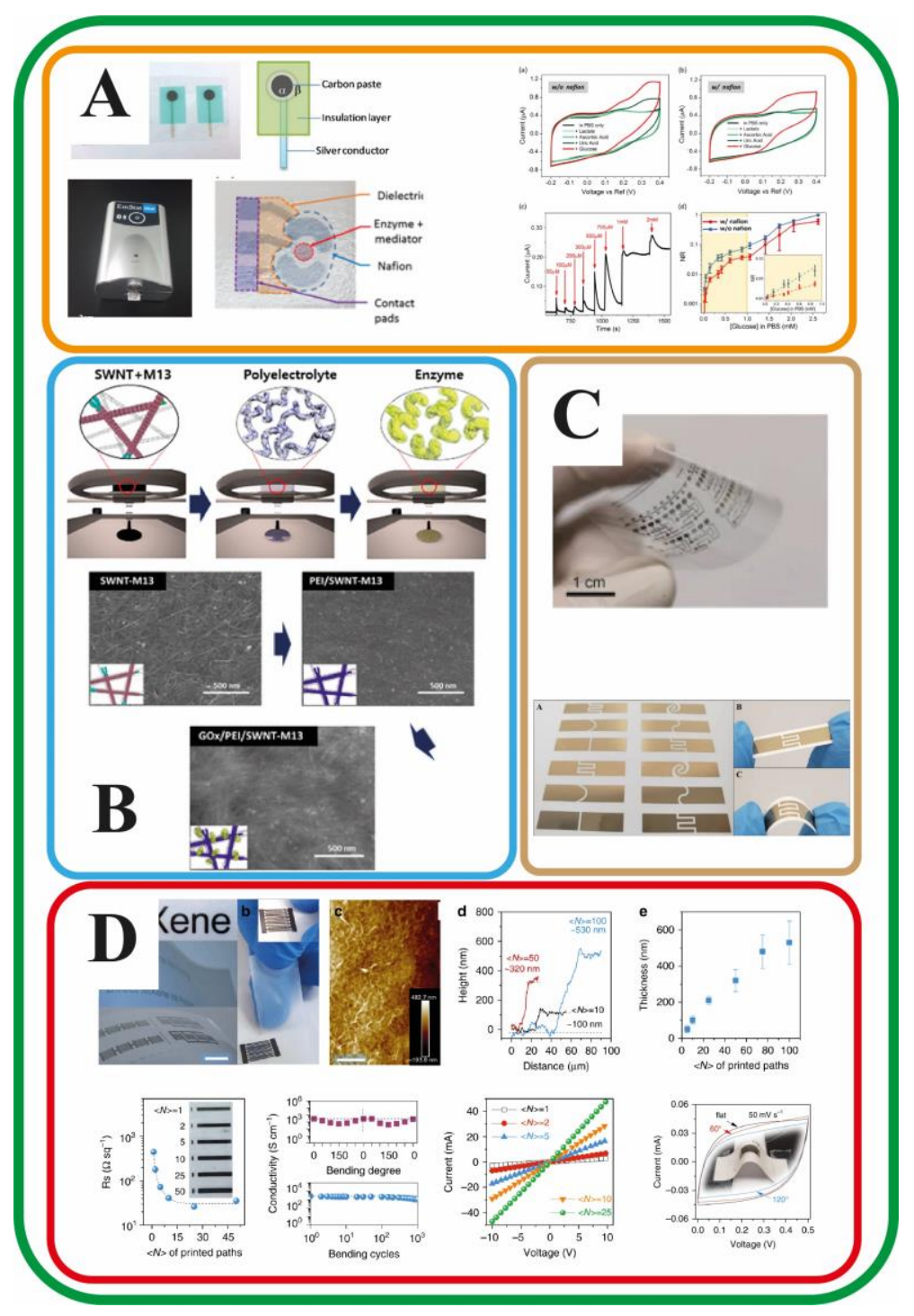

Figure 5. IJP-based electrodes. (A) and (B) demonstrate that multiple layers of the working electrode can be fabricated via IJP. ${ }^{[270,274]}(\mathbf{C})$ showcases printed electrodes that can be achieved, ${ }^{[275,276]}$ whereas (D) provides electrochemical performance as a function of bending, along with other parameters. ${ }^{[95]}$ Figures were adapted and printed with permission from Elsevier B.V., Springer Nature and American Chemical Society.

Similar to DIW, post-processing usually entails evaporating the solvents or sintering of metallic nanoparticles. Graphene oxide inks can be further subjected to electrochemical reduction to obtain reduced graphene oxides, which can be employed instead of thermal reduction to avoid damage to substrates with low thermal stability. Here, the same conditions reported for electrochemical activation are also determinants, such as reduction time and voltage. For example, a recent study revealed that a $45 \%$ increase in electrochemical activity 
can be obtained when IJP-printed graphene oxide traces are subsequently electrochemically reduced. ${ }^{[277]}$ The result was an electrode with a limit of detection of $1.6 \mathrm{ng} / \mathrm{ml}$ for HT-2 toxins that is comparable to high performance liquid chromatography, which is a widely used standard quantification method. Overall, the recent progress made in IJP-fabricated electrodes demonstrate that the technology can exceed the current limitations of traditional fabrication techniques.

\subsubsection{Aerosol Jet Printing}

Besides DIW and IJP, AJP is also demonstrating promise for fabricating electrodes. Because of high printing resolution, can process materials with higher viscosities and larger particle sizes, which makes AJP an ideal technology for fabricating 3D structures. In addition, the technology can produce sophisticated structures, which further expands the applications of direct writing techniques. ${ }^{[278]}$ For example, AJP can produce microelectrode array consisting of PEDOT:PSS microneedles, with a diameter and height of 10 and $33 \mu \mathrm{m}$, respectively. ${ }^{[279]}$ As anticipated, additional additives are required to achieve a standing structure with this technology, which included MWCNT for mechanical stability, and both (3-glycidyloxypropyl) trimethosilane and carboxymethyl cellulose to ensure stability, which acted as an adhesion promoter and emulsifier, respectively. The stability of the electrode was further verified under aqueous solution, which is pivotal for the electrode to be suitable for electrochemical analysis. The conductivity was measured as $323 \mathrm{~S} / \mathrm{m}$. The appeal of developing microneedle arrays stems from the need to obtain live measurements. Microneedles are able to penetrate the skin and access the interstitial fluid. Previous studies have reported good correlation between the interstitial fluid and intravenous levels for bioanalytes of interest. Thus, the goal is to use microneedles for minimally-invasive continuous monitoring. Apart from this, recent work has demonstrated AJP can be used to detect glucose, cytokines, proteins, and histamine. ${ }^{[21,280-282]}$ 
Electrodes with trace widths as low as $15 \mu \mathrm{m}$ can be achieved, and as expected, both the printing speed and number of deposited layers affect the dimensions of the traces, and consequently the electrochemical properties. ${ }^{[283]}$ These studies demonstrate that AJP is a versatile AM technology, capable of achieving high resolution electrodes with complex geometries. Overall, direct writing technologies provide promising electrodes with high flexibility, scalability, variability and suitable for animal studies. These AM technologies are capable of one-step printing both the conductive and biorecognition layers, and hence will facilitate the electrode fabrication process. For electrode preparation that necessitates annealing, future work should find a solution to accelerate the post-processing stage (e.g. annealing) in order to realize the goal of on-demand printing, such as flash annealing that can

anneal in the order of milliseconds. ${ }^{[284,285]}$, and subsequently elucidate the effects of flash annealing on the final electrochemical performance.

\subsection{Vat Polymerization}

SLA and DLP are also widely used AM techniques, owing to their compactness and low instrument cost. Both technologies are capable of achieving resolutions greater than FDM and SLM, which makes them ideal for fabricating microstructures. ${ }^{[286]}$ Both SLA and DLP belong to a group of AM technologies collectively referred to as vat polymerization. There are slight differences between SLA and DLP in their fabrication approach. Both use liquid resins to form a solid 3D structure via light. At its simplest, the resin is comprised of photo-curable monomers and a photoinitiator. When the resin is excited by light, the photoinitiators initiate the crosslinking of the photo-curable monomers to form photopolymers. This process happens layerby-layer until the desired geometry is obtained. Following this, the object is removed from the printer and is washed using a solvent to remove any uncured monomers. Thereafter the object 
is placed in a curing oven to impart more structural stability. SLA uses a laser that 'writes' the shape of the object at each layer; whereas DLP projects the entire layer shape at once. ${ }^{[287]}$

SLA and DLP have also been investigated as potential candidates for electrode fabrication. Similar to FDM, the feedstock for SLA/DLP is non-conductive, and thus conductive fillers are required. Polyethylene glycol diacrylate (PEGDA), a frequently used photopolymer, was blended with PEDOT:PSS, where a resistivity of $\sim 10 \mathrm{Ohms} \bullet \mathrm{cm}^{[288]}$ was obtained. MWCNT were also blended with PEGDA, albeit obtaining a higher resistivity of 2.5 $\mathrm{x} 10^{3} \mathrm{Ohms} \cdot \mathrm{cm}^{\left[{ }^{[289]}\right.}$ For fabricating electrodes, a utilized approach is to thermally post-process the printed parts under inert gas, which results in a carbonaceous material. This can be done using commercial resins, as performed by Rezaei et al. (2020) (Figure 6 (A)), or an in-house formulation. Indeed, the concept is to obtain a conductive part whilst taking advantage of vatpolymerizations remarkable resolution. However, this strategy does not appear to work for all commercial resins, with some printed parts collapsing following pyrolysis. ${ }^{[290]}$ Fundamentally, it depends on the chemical composition of the starting resin. As expected, pyrolysis will induce both mass loss and linear shrinkage, which can be as high as $95 \%$ and $60 \%$, respectively, and will depend on both the pyrolysis temperature and heating rate. BET analysis revealed that faster heating rates produce a greater number of micropores, possibly due to less time for degassing. Thus, to facilitate degassing, and by extension obtain a free-standing structure, incorporating open and connected pores in the original design is recommended. Additionally, faster heating rate also produces a rougher surface, which consequently improves the electrochemical properties of the electrode.

SLA can also be used to fabricate microneedle arrays using the pyrolysis strategy (Figure $6(\mathbf{B}) \&(\mathbf{C})$ ). One major concern with photopolymers is their toxicity, and hence a microneedle fabricated using photopolymers will not be a viable approach until these biocompatibility obstacles are addressed. 


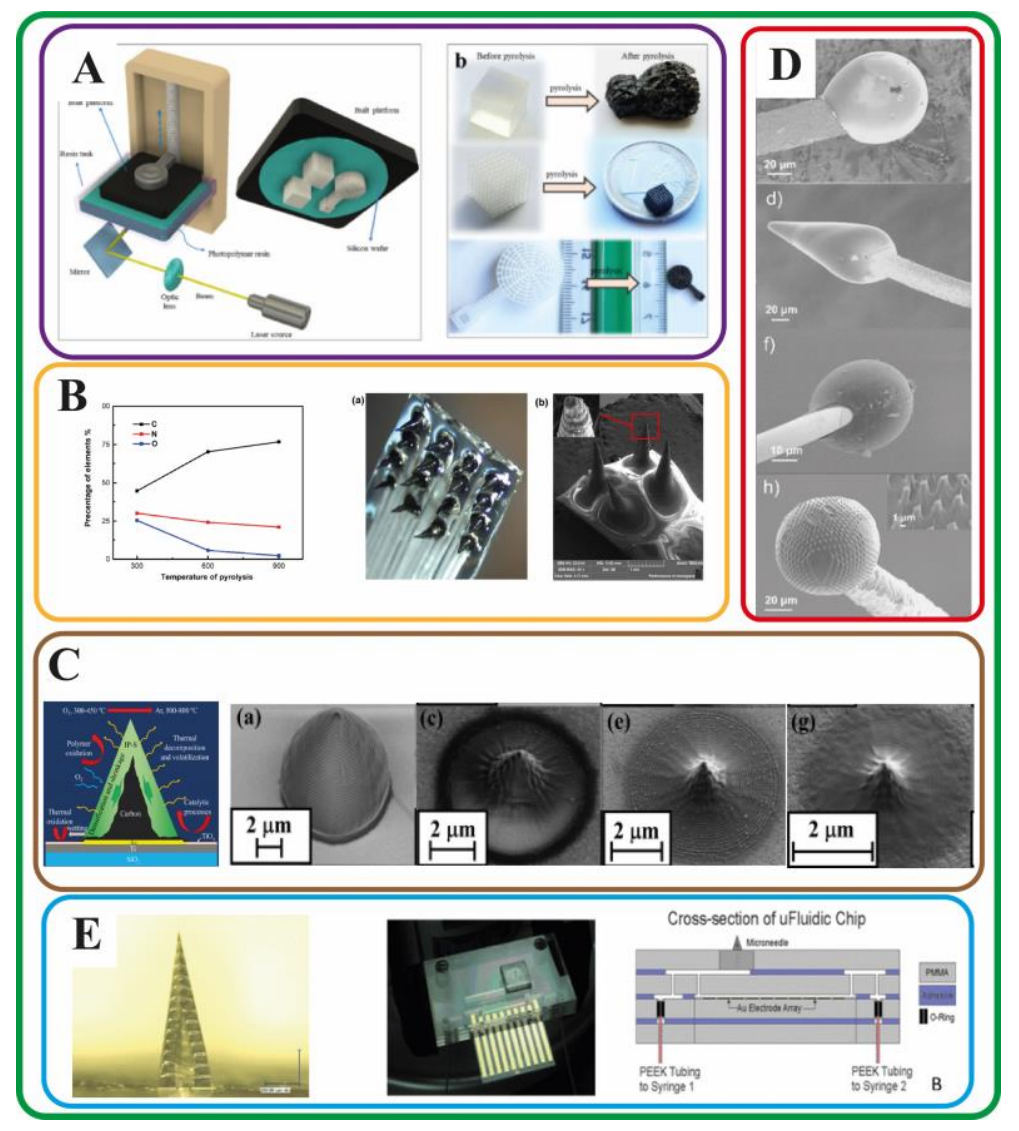

Figure 6. Vat-polymerization electrodes require pyrolysis to impart conductivity whilst maintaining biocompatibility. (A) depicts the change in SLA prints of commercial resin following pyrolysis. ${ }^{[290]}$ (B) demonstrates that microneedle structures are feasible with this strategy, whereas (C) are SEM micrographs illustrating the shrinkage of the microneedles with increasing pyrolysis temperature. ${ }^{[291,292]} 2 \mathrm{PP}$ has also been used to develop both (D) conductive elements and (E) microneedle with an inlet port for extracting interstitial fluid. ${ }^{[293,294]}$ Figures were adapted and printed with permission from Elsevier B.V., John Wiley and Sons, American Vacuum Society, and IEEE.

On the other hand, carbon-based microneedles are a biocompatible alternative that can produced from pyrolyzing SLA prints. Prior work successfully fabricated microneedles with a height of $1.5 \mathrm{~mm}$ and an exposure tip of $20 \mu \mathrm{m} .{ }^{[291]}$ Compared to commercial $\mathrm{Pt}$ microelectrodes, the carbonaceous microneedles possessed a lower impedance, and during in vivo measurements, resulted in a higher signal-to-noise ratio of 50.73, compared to 20.15 for the Pt microelectrode. 
The pyrolysis step is not always straightforward, and can result in microneedles with distorted shapes if processing measures are not implemented. For example, the polymer can soften during pyrolysis, which will cause the microneedles to lose their shape fidelity. Another issue is the accumulation of carbon into concentrated areas on the microneedle, which when excessive will prevent the gas from escaping. These issues can be resolved by (i) pre-heating the polymer to its glass transition temperature, which prevented it from softening; and (ii) positioning the $3 \mathrm{D}$ print in such a manner that it prevents undesirable carbon accumulation. In a recent study, the prints were inverted inside of the furnace, which was found to limit gas evolution. Other processing conditions will need to be factored if a multi-material design is to be pyrolysed, as different materials shrink at different rates, as well as consideration to the volatiles produced and if they will unwantedly react with the carbonaceous structure. ${ }^{[292]}$ What is also lacking is mechanical testing of the pyrolyzed structures, and whether they have sufficient mechanical strength to penetrate human skin. Moreover, further analyses will need to confirm electrochemical stability over prolonged measurement periods.

Besides SLA and DLP, a cutting-edge technology in vat polymerization called twophoton polymerization (2PP) has also been used to fabricate free-standing conical and spherical carbon electrode using the printing-pyrolysis strategy (Figure $6(\mathbf{D})) \cdot{ }^{[293]} 2 \mathrm{PP}$ is also capable of printing microneedles with a feature size of $0.5 \mu$ mean be attained, ${ }^{[295]}$ which is challenging for either SLA/DLP and AJP. For instance, previous work devised a microneedle with a pore that served as an inlet into a microfluidic chip (Figure $6(\mathbf{E})) .{ }^{[294]}$ The aim was to use the device to sample multiple biomarkers within interstitial fluid, where the microneedle was used to penetrate the skin, and subsequently extract the desired fluid. The microfluidic device itself consisted of eight working electrodes, where a manifold was used to regulate fluid flow. The study itself was a proof-of-concept that resembles the Freestyle Libre glucose sensor that is commercially available, but more work is needed to validate the novel design, including 
interference studies. The drawbacks of $2 \mathrm{PP}$ is that the instrument itself demands comparatively high capital costs, and that although sub-micron resolutions can be obtained, this is at the expense of speed. Until both obstacles are addressed, it limits the technology from being used for on-demand clinical applications.

\subsection{Selective Laser Sintering and Melting}

SLS and SLM are another group of AM technology that have been used to fabricate electrodes. Both technologies can fabricate polymeric, ceramic and metallic structures. ${ }^{[296-299]}$ They both use lasers to weld particulate matter into a monolithic structure; upon receiving the sliced image, the powder bed is scanned and processed on the pre-designed CAD model. Once the laser has scanned the first layer, a fresh layer of powder is placed, and the processes is repeated again, until the 3D structure is obtained. The difference between SLM and SLS is that melting of the powder is achieved in the former, and sintering is needed for the latter. Sintering is a welding process achieved without complete melting of the material. This is achieved by atoms from neighboring particles diffusing across and weld the particles together. Both techniques have a post-processing step entails removing unsintered or un-melted powder. These methods offer a range of advantages including near-net shape production, lower production times and complex shape designs. ${ }^{[300]}$ The resolution of SLS/SLM are approx. $10-100 \mu \mathrm{m}$, depending on factors such as laser spot size, writing speed, and particle size. ${ }^{[301,302]}$

SLM and SLS have been demonstrated to produce electrodes that, albeit not applied as electrochemical biosensors, validate their potential. Serkov et al. (2019) used SLS to form tindoped indium oxide thin films on a PET substrate. ${ }^{[303]}$ Their approach precluded the need for high temperature sintering that would otherwise damage PET. Zacharatos et al. (2018) used SLS for the fabrication of AgNPs onto a polyethylene napthalate substrate, which exhibited a glass transition and melting temperature of 121 and $269^{\circ} \mathrm{C}$, respectively. ${ }^{[304]}$ The study sought- 
out to examine the effect of laser sintering parameters on the conductivity of the composite, and reported resistivity as low as $9.44 \pm 0.5 \mu \Omega \bullet \mathrm{cm}$. Delamination studies further revealed the sintered AgNPs were sufficiently adhered onto the PEN substrate. It is worth remarking that the costings of this novel approach are yet to be determined. Kwon et al. (2016) demonstrated that selective laser sintering can be used to fabricate CuNPs onto a polymer substrate, thereby fabricating a conductive, flexible material, and therewithal transparent. ${ }^{[305]}$ The approach demonstrated that $\mathrm{Cu}$, which is susceptible to oxidation, can be sintered with remarkable oxidation suppression condition, rapidly, and low annealing temperatures. The applications for SLM have focused in demonstrating the technology can achieve complex electrode designs. ${ }^{[306]}$ In particular, metallic helical electrodes for detecting paracetamol, dopamine, ${ }^{[307]}$ nerve agents, ${ }^{[308]}$ ascorbic and uric acid. ${ }^{[29]}$ In the latter study, SLM-printed electrodes were found to possess high accuracy, and had a superior performance compared to a standard $3 \mathrm{~mm}$ glassy carbon electrode.

In addition to their excellent electrochemical performance, both SLS and SLM produce prints with good surface finish, high mechanical stability and can achieve high throughput printing. However, a good surface finish is not always desirable since rough surfaces are able to facilitate the binding of bioanalytes. Moreover, both technologies consume large amounts of powder, that if oxidized will need to be disposed of. Both metal SLS and SLM printing is targeted at large-sized objects, up to the order of meters, and hence the instruments occupy a large laboratory footprint compared to AM technologies. Moreover, the cost of a machine is more expensive than other AM technologies. Hence, it is unlikely that SLS and SLM are suitable for on-demand printing in clinical settings. 


\subsection{Auxiliary Components}

AM has multiple benefits when printing sensors in addition to printing conductive electrodes. Applications include simple use of printing for potentiostat casings, where bespoke designs can be made to minimize component volume and weight. ${ }^{[309]}$ Fluidic chambers were also developed to facilitate sensing capabilities or rapidly prototype mimicked continuous flow chambers. ${ }^{[310-320]}$ These can be cheaply printed and on-demand, rather than purchasing them in bulks, which collectively minimizes costs. Another example was illustrated where AM was involved in developing a cheaper alternative to the costly reference electrodes. ${ }^{[321]}$

Forward-looking applications consider the potential integration of biosensors with wearable technologies, where AM was used to develop personalized casings. ${ }^{[322,323]}$ It is worth remarking that personalized wearables, particularly form-fitting, may play a crucial part in improving patient acceptance and compliance. In a different demonstration for smart devices, Bandodkar et al. (2018) developed an innovative integrated smartphone platform for detecting glucose, leveraging the fact that smartphones are ubiquitously used across society. ${ }^{[324]}$ The phone case was 3D printed, but more notably, so was a stylus for housing enzyme pellets, which were deposited onto unfunctionalized electrodes when needed. The rationale for the latter was to preserve enzyme function when not required (i.e. when not actively measuring glucose), and thereby extending the longevity of the biorecognition element by mitigating enzyme leaching and degradation. Other studies have also developed smartphone adapters for portable sensors. ${ }^{[325,326]}$ These examples demonstrate AM practicality to circumvent design bottlenecks, with the potential to holistically fabricate all aspects of the biosensor. Auxiliary components fabricated are portrayed in Figure 7. 


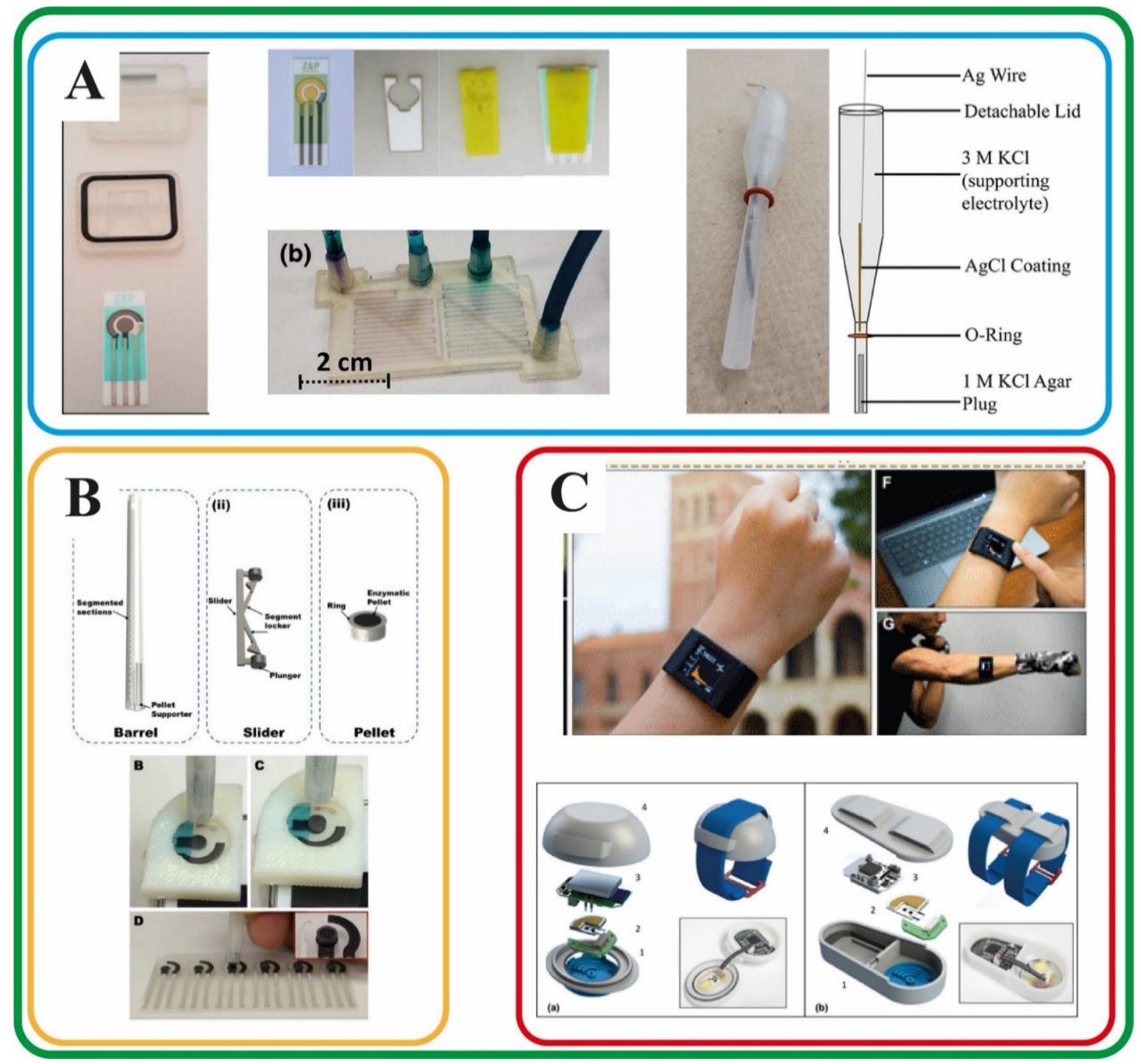

Figure 7. Auxiliary components fabricated by AM. Auxiliary components range from fabricating laboratory-grade components to facilitate analysis (A), to wearable technologies (B) \& (C) fitted to consumers' needs. ${ }^{[310,311,321-324,327]}$ Figures were adapted and printed with permission from Elsevier B.V., MDPI, American Chemical Society, and AAAS Publication.

\subsection{Additive Manufacturable Circuit Boards}

Remarkably AM has also been used to fabricate a circuit board. An all-printed circuit and electrode were fashioned using AJP, which was lightweight $(<5 \mathrm{~g})$ and thin $(<2 \mathrm{~mm})$ enough for wearable applications (Figure $8(\mathbf{A})$ ). ${ }^{[328]}$ The circuit board, printed using a combination of polyimide and silver for the insulating and conductive elements, respectively, showed good mechanical compliance to various flexible applications. The Ag traces were phototonically 
sintered at $2 \mathrm{kV} / 2 \mathrm{~ms}$ for five times, for rapid sintering. Functionalized graphene was subsequently printed on silver traces to act as an oxidative barrier, and prevent the solder from consuming the silver (Figure 8 (A)). Furthermore, SEM micrographs illustrated the uniformity of the layer-by-layer structure, and thereby confirming the capability of AJP to enable reliable deposition of multiple materials sequentially. The electronic components were soldered onto the device, including a wireless circuit for external communication. The electrode was fabricated using functionalized graphene, and it too which also displayed mechanical compliance to various flexing motions. The circuit and electrode were used for electromyogram analysis, and one envisages that all-printed circuit and electrode via AJP can be made for wearable electrochemical biosensors.

Mechanically-actuated DIW has also been used to print circuit boards (Figure 8 (B)). ${ }^{[263]}$ Again, the AM technology was used to print both the electrodes and printed circuit board, which were fabricated with a highly viscous AgNP ink. The aim was to detect lactate, which was achieved with a detection region of 0-20 mM. Another technique that has been demonstrated to print electrochemical devices is fiber encapsulation additive manufacturing (FEAM). ${ }^{[329]}$ However, the technology is yet to be applied as a biosensor due to its high cost and complexity. ${ }^{[330]}$ 


\section{A}
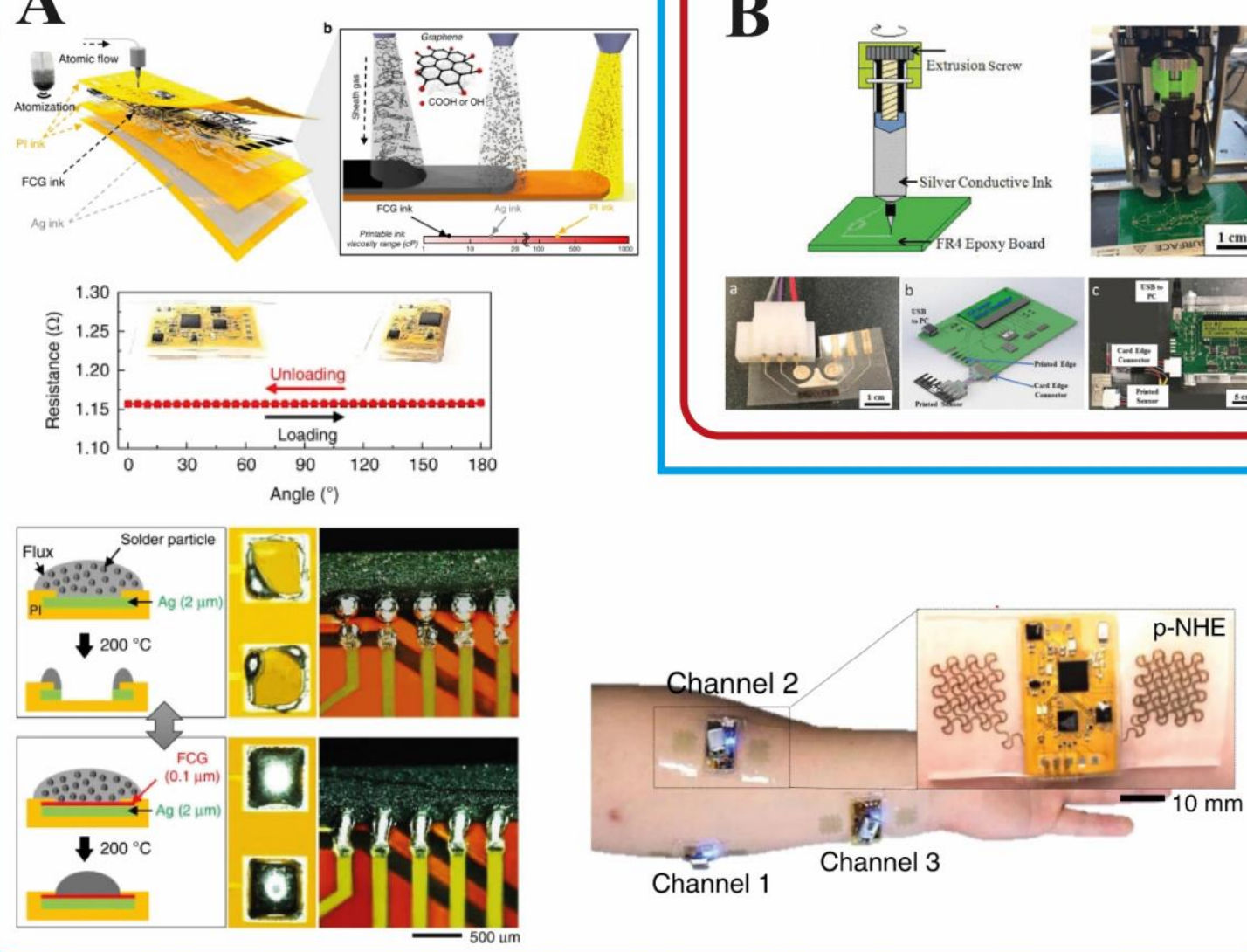

Figure 8. AM for fabricating circuit boards using (A) AJP and (B) DIW. ${ }^{[263,328]}$ In both examples, AM was used to print the conductive tracks on the printed circuit board and the electrodes. Figures were adapted and printed with permission from IEEE, and Springer Nature.

\section{Prospect and Future Direction of AM electrodes}

In this progress report, several AM techniques, their strategies, and the different conductive materials were reviewed. The field is still in its infancy, yet significant developments have already been made, including the fabrication of electrodes with either comparable or superior electrochemical performances to that of traditional electrodes. It is also evident that as the wider biosensor field is transitioning toward wearables, techniques such as AJP are maintaining similar progress to that of traditional fabrication methods. However, AM techniques have with 
the added benefits of producing low-cost electrodes, fast throughput and multi-material fabrication, which is a step toward automating electrode design. Equally important innovations are studies that have demonstrated the fabrication of both a circuit board and electrode from the same technology, which has seldom been achieved with traditional techniques. As the field matures, one envisages that further advancements will be made to improve the performances of AM-based electrodes.

The primary focus of the field has centered on printing conductive electrodes, which has been achieved by all techniques described in this review. It is clear from the highlights made in Section 3 that emerging materials are yet to be investigated. For example, the majority of FDM conductive filaments either use metallic or carbonaceous fillers, and hence more work should be devoted toward exploring quantum dots or MXene to determine their electrochemical properties when processed via FDM. Furthermore, there will be a reliance in new materials discovered (carbon ceramic electrodes) ${ }^{[331]}$ to further improve sensing performance. One aspect that has not been thoroughly explored is the printing of biorecognition elements, where most research remains reliant on drop-casting. For example, SLA has been used to immobilize enzymes, which remained active following the fabrication process. ${ }^{[332]}$ Exploiting the spatial resolution of SLA can be used to produce honeycomb structures to further maximize surface area, and consequently enzyme activity. Moreover, MIP are widely used to enhance electrode selectivity, yet AM-based MIP are underutilized. As mentioned, AM can be used to produce complex structures with large surface area to further improve selectivity, and in a controllable manner. Furthermore, long-term biocompatibility studies will need to be performed to ensure that AM-electrodes are suitable for long-term applications, such as in the case of microneedles for continuous monitoring.

From the progress made thus far, it is clear that most of the studies employed some form of post-processing. Some of the more involved processes, such as those needing a furnace 
for pyrolysis, could limit the potential of some AM technologies from being utilized on-site. Hence, consideration should be given as to how the overall process can be streamlined to achieve on-demand production. There are other AM technologies that are yet to be explored that could lead to new innovations. An example of this is microscale-SLS, with sub $5 \mu \mathrm{m}$ resolution, rapid throughput of over $60 \mathrm{~mm}^{3} / \mathrm{h}$, and can print $\mathrm{Ag}$ nanoparticles with conductivity in the same magnitude as bulk silver. ${ }^{[301]}$ Another recent innovation is AM for 3D nano-architected metals, which has the potential to fabricate conductive parts without sacrificing nano-scale features. As mentioned, sintering of nanoparticles results in loss of nano features, which consequently reduces the electron transfer and redox mediating properties. ${ }^{[31]}$ Nonetheless, some of the existing technologies reported herein, such as DIW and AJP, have shown great prospect to address many of the limitations observed by traditional fabrication techniques.

\section{Conclusion}

In an era where affordable healthcare and precision medicine are desired, the choice of material and fabrication technology will underpin the achievements of such goals. Fortunately, there is an abundance of materials to select from, including conductive polymers, metals and carbonaceous materials. As discussed herein, compositing materials offer a strategic approach to imparting conductivity to materials that are fabrication friendly but lack conductivity. AM opens the opportunity of attaining a rapid point-of-care diagnostic tool. Despite the technology being in its infancy, AM has demonstrated that cost-effective and complex designs can be attained, with electrochemical performances that exceed traditionally-fabricated electrodes. In addition, recent progress has demonstrated that AM can be used to print multiple aspects of EC biosensors, including functioning circuit boards. The current progress has demonstrated that AM provides several advantages for developing electrodes for research purposes, and further advancements are needed to determine suitability for clinical applications. 


\section{References}

[1] M. Bacco, A. Berton, E. Ferro, C. Gennaro, A. Gotta, S. Matteoli, F. Paonessa, M. Ruggeri, G. Virone, A. Zanella, In 2018 IoT Vertical and Topical Summit on Agriculture - Tuscany (IOT Tuscany); 2018; pp. 1-6.

[2] R. H. Czichy, Aerospace Sensors. Sensors Set 1995, 365-411.

[3] D. Dornfeld, Fundamentals: Principles of Sensors in Manufacturing. Sensors Manuf. 2001, 6-23.

[4] S. Leonardo, A. Toldrà, M. Campàs, Recent Adv. Anal. Mar. Toxins, Compr. Anal. Chem. 2017, 303.

[5] S. Kumar, S. D. Bukkitgar, S. Singh, Pratibha, V. Singh, K. R. Reddy, N. P. Shetti, C. Venkata Reddy, V. Sadhu, S. Naveen, ChemistrySelect 2019, 4, 5322.

[6] O. Elijah, T. A. Rahman, I. Orikumhi, C. Y. Leow, M. N. Hindia, IEEE Internet Things J. 2018, 5, 3758.

[7] O. Simoska, K. J. Stevenson, Analyst 2019, 144, 6461.

[8] B. Reddy, U. Hassan, C. Seymour, D. C. Angus, T. S. Isbell, K. White, W. Weir, L. Yeh, A. Vincent, R. Bashir, Nat. Biomed. Eng. 2018, 2, 640.

[9] P. Kassal, M. D. Steinberg, I. M. Steinberg, Sensors Actuators B Chem. 2018, 266, 228.

[10] S. Aiassa, S. Carrara, D. Demarchi, IEEE Sensors Lett. 2019, 3, 1.

[11] P. P. Ray, D. Dash, N. Kumar, Comput. Commun. 2020, 160, 111.

[12] V. Scognamiglio, F. Arduini, TrAC Trends Anal. Chem. 2019, 120, 115642.

[13] H. Hafezi, T. L. Robertson, G. D. Moon, K. Au-Yeung, M. J. Zdeblick, G. M. Savage, IEEE Trans. Biomed. Eng. 2015, 62, 99.

[14] N. Bhalla, P. Jolly, N. Formisano, P. Estrela, Essays Biochem. 2016, 60, 1.

[15] M. A. Sakr, K. Elgammal, A. Delin, M. Serry, Sensors 2020, 20, 145.

[16] A. Uzunoglu, D. A. Kose, E. Gokmese, F. Gokmese, J. Clust. Sci. 2020, 31, 231.

[17] E. A. Ashley, Nat. Rev. Genet. 2016, 17, 507.

[18] T. Pollard, J. Ong, A. Goyanes, S. Gaisford, M. Elbadawi, A. W. Basit, Drug Discov. Today 2020, In press.

[19] P. Niu, M. Gich, C. Fernández-Sánchez, A. Roig, Sol-Gel Nanocomposites for Electrochemical Sensor Applications. Sol-Gel Handb. 2015, 1413-1434.

[20] H. Yuk, B. Lu, S. Lin, K. Qu, J. Xu, J. Luo, X. Zhao, Nat. Commun. 2020, 11, 1604.

[21] K. Parate, S. V Rangnekar, D. Jing, D. L. Mendivelso-Perez, S. Ding, E. B. Secor, E. A. Smith, J. M. Hostetter, M. C. Hersam, J. C. Claussen, ACS Appl. Mater. Interfaces 2020, $12,8592$.

[22] Y. Dong, S. Wang, Y. Ke, L. Ding, X. Zeng, S. Magdassi, Y. Long, Adv. Mater. Technol. 2020, 5, 2000034.

[23] T. D. Ngo, A. Kashani, G. Imbalzano, K. T. Q. Nguyen, D. Hui, Compos. Part B Eng. 2018, $143,172$.

[24] S. J. Trenfield, A. Awad, C. M. Madla, G. B. Hatton, J. Firth, A. Goyanes, S. Gaisford, A. W. Basit, Expert Opin. Drug Deliv. 2019, 16, 1081.

[25] R. Hashemi Sanatgar, C. Campagne, V. Nierstrasz, Appl. Surf. Sci. 2017, 403, 551.

[26] A. H. Espera, J. R. C. Dizon, Q. Chen, R. C. Advincula, Prog. Addit. Manuf. 2019, 4, 245.

[27] M. Elbadawi, B. Muñiz Castro, F. K. H. Gavins, J. J. Ong, S. Gaisford, G. Pérez, A. W. Basit, P. Cabalar, A. Goyanes, Int. J. Pharm. 2020, 590, 119837.

[28] L. Scime, J. Beuth, Addit. Manuf. 2018, 19, 114.

[29] E. H. Z. Ho, A. Ambrosi, M. Pumera, Appl. Mater. Today 2018, 12, 43.

[30] T. Kant, K. Shrivas, V. Ganesan, Y. K. Mahipal, R. Devi, M. K. Deb, R. Shankar, 
Microchem. J. 2020, 155, 104687.

[31] A. Vyatskikh, S. Delalande, A. Kudo, X. Zhang, C. M. Portela, J. R. Greer, Nat. Commun. 2018, 9, 593.

[32] J. G. van den Tweel, C. R. Taylor, Virchows Arch. 2010, 457, 3.

[33] G. A. Posthuma-Trumpie, J. Korf, A. van Amerongen, Anal. Bioanal. Chem. 2009, 393, 569.

[34] C. V. Raman, 1928.

[35] I. Notingher, Sensors 2007, 7, 1343.

[36] K. E. Shafer-Peltier, C. L. Haynes, M. R. Glucksberg, R. P. Van Duyne, J. Am. Chem. Soc. 2003, 125, 588.

[37] F. Severcan, P. I. Haris, Vibrational spectroscopy in diagnosis and screening; IOS Press, 2012; Vol. 6.

[38] M. N. Mohammed, H. Syamsudin, S. Al-Zubaidi, R. R. AKS, E. Yusuf, Int. J. Psychosoc. Rehabil. 2020, 24.

[39] D. S. W. Ting, L. Carin, V. Dzau, T. Y. Wong, Nat. Med. 2020, 26, 459.

[40] S. Swayamsiddha, C. Mohanty, Diabetes Metab. Syndr. Clin. Res. Rev. 2020, 14, 911.

[41] V. Gaudin, Biosens. Bioelectron. 2017, 90, 363.

[42] E. T. S. G. da Silva, D. E. P. Souto, J. T. C. Barragan, J. de F. Giarola, A. C. M. de Moraes, L. T. Kubota, ChemElectroChem 2017, 4, 778.

[43] S. M. Yoo, S. Y. Lee, Trends Biotechnol. 2016, 34, 7.

[44] S. A. Lim, M. U. Ahmed, In Food Biosensors; The Royal Society of Chemistry, 2017; pp. 1-21.

[45] P. Kubáň, P. C. Hauser, Electrophoresis 2009, 30, 3305.

[46] D. Sarkar, W. Liu, X. Xie, A. C. Anselmo, S. Mitragotri, K. Banerjee, ACS Nano 2014, 8, 3992.

[47] J. Zhao, G. Chen, Li, G. B. T.-N.-I. B. for P. A. with C. A., Ed.; Elsevier, 2019; pp. xviixlvii.

[48] W. Gao, S. Emaminejad, H. Y. Y. Nyein, S. Challa, K. Chen, A. Peck, H. M. Fahad, H. Ota, H. Shiraki, D. Kiriya, D.-H. Lien, G. A. Brooks, R. W. Davis, A. Javey, Nature 2016, 529, 509.

[49] M. A. Morales, J. M. Halpern, Bioconjug. Chem. 2018, 29, 3231.

[50] J. H. T. Luong, J. D. Glennon, A. Gedanken, S. K. Vashist, Microchim. Acta 2017, 184, 369.

[51] K. Manoli, M. Magliulo, M. Y. Mulla, M. Singh, L. Sabbatini, G. Palazzo, L. Torsi, Angew. Chemie Int. Ed. 2015, 54, 12562.

[52] W. Suginta, P. Khunkaewla, A. Schulte, Chem. Rev. 2013, 113, 5458.

[53] E. M. I. M. Ekanayake, D. M. G. Preethichandra, K. Kaneto, Sensors Actuators B Chem. 2008, 132, 166.

[54] N. Mansouri, A. A. Babadi, S. Bagheri, S. B. A. Hamid, Int. J. Hydrogen Energy 2017, 42, 1337.

[55] Y. Ji, J. Liu, X. Liu, M. M. F. Yuen, X.-Z. Fu, Y. Yang, R. Sun, C.-P. Wong, Electrochim. Acta 2017, 248, 299.

[56] K. Lu, Science (80-. ). 2010, 328, 319 LP.

[57] H. F. Li, Y. F. Zheng, Acta Biomater. 2016, 36, 1.

[58] J. P. Chu, T.-Y. Liu, C.-L. Li, C.-H. Wang, J. S. C. Jang, M.-J. Chen, S.-H. Chang, W.C. Huang, Thin Solid Films 2014, 561, 102.

[59] M. M. Khan, K. M. Deen, W. Haider, J. Non. Cryst. Solids 2019, 523, 119544.

[60] D. V Louzguine-Luzgin, V. I. Polkin, Russ. J. Non-Ferrous Met. 2017, 58, 80.

[61] N. Li, J. Zhang, W. Xing, D. Ouyang, L. Liu, Mater. Des. 2018, 143, 285.

[62] E. R. Kinser, J. Padmanabhan, R. Yu, S. L. Corona, J. Li, S. Vaddiraju, A. Legassey, A. 
Loye, J. Balestrini, D. A. Solly, J. Schroers, A. D. Taylor, F. Papadimitrakopoulos, R. I. Herzog, T. R. Kyriakides, ACS Sensors 2017, 2, 1779.

[63] D. Foxx, E. E. Kalu, Electrochem. commun. 2007, 9, 584.

[64] N. Dimcheva, Curr. Opin. Electrochem. 2020, 19, 35.

[65] J. Lu, I. Do, L. T. Drzal, R. M. Worden, I. Lee, ACS Nano 2008, 2, 1825.

[66] B. T. Anto, S. Sivaramakrishnan, L.-L. Chua, P. K. H. Ho, Adv. Funct. Mater. 2010, 20, 296.

[67] Y. Liu, Y. Wang, R. O. Claus, Chem. Phys. Lett. 1998, 298, 315.

[68] X.-Y. Zhang, J.-J. Xu, J.-Y. Wu, F. Shan, X.-D. Ma, Y.-Z. Chen, T. Zhang, RSC Adv. 2017, 7, 8 .

[69] J. C. Tan, H. Y. Low, Addit. Manuf. 2018, 23, 294.

[70] J. Li, Z. Shen, X. Chen, S. Yang, W. Zhou, M. Wang, L. Wang, Q. Kou, Y. Liu, Q. Li, Z. Xu, Y. Chang, S. Zhang, F. Li, Nat. Mater. 2020.

[71] H. L. Tuller, In Ceramic Materials for Electronics; CRC Press USA, 2004; pp. 87-140.

[72] G. Hautier, A. Miglio, G. Ceder, G.-M. Rignanese, X. Gonze, Nat. Commun. 2013, 4, 2292.

[73] C. Diao, H. Liu, H. Zheng, Z. Yao, J. Iqbal, M. Cao, H. Hao, J. Alloys Compd. 2018, $765,362$.

[74] S. Ye, A. R. Rathmell, Z. Chen, I. E. Stewart, B. J. Wiley, Adv. Mater. 2014, 26, 6670.

[75] E. B. Aydın, M. K. Sezgintürk, TrAC Trends Anal. Chem. 2017, 97, 309.

[76] Z. Chen, X. Qin, T. Zhou, X. Wu, S. Shao, M. Xie, Z. Cui, J. Mater. Chem. C 2015, 3, 11464.

[77] M. Hwang, B. Jeong, J. Moon, S.-K. Chun, J. Kim, Mater. Sci. Eng. B 2011, 176, 1128.

[78] H.-K. Kim, I.-K. You, J. B. Koo, S.-H. Kim, Surf. Coatings Technol. 2012, 211, 33.

[79] J.-A. Jeong, J. Kim, H.-K. Kim, Sol. Energy Mater. Sol. Cells 2011, 95, 1974.

[80] F. Shao, Q. Wan, J. Phys. D. Appl. Phys. 2019, 52, 143002.

[81] M. Fang, A. Aristov, K. V Rao, A. V Kabashin, L. Belova, RSC Adv. 2013, 3, 19501.

[82] A. A. Ansari, M. Alhoshan, M. S. Alsalhi, A. S. Aldwayyan, Biosensors 2010, 302.

[83] B. Liu, J. Liu, TrAC Trends Anal. Chem. 2019, 121, 115690.

[84] C. Magnus, D. Cooper, J. Sharp, W. M. Rainforth, Wear 2019, 438-439, 203013.

[85] N. C. Ghosh, S. P. Harimkar, Low, I. M. B. T.-A. in S. and T. of M. P., Ed.; Woodhead Publishing, 2012; pp. 47-80.

[86] X. Fan, Y. Ma, X. Dang, Y. Cai, Materials (Basel). 2020, 13, 328.

[87] M. Magnuson, M. Mattesini, Thin Solid Films 2017, 621, 108.

[88] M. A. Hadi, S.-R. G. Christopoulos, S. H. Naqib, A. Chroneos, M. E. Fitzpatrick, A. K. M. A. Islam, J. Alloys Compd. 2018, 748, 804.

[89] J. Jimmy, B. Kandasubramanian, Eur. Polym. J. 2020, 122, 109367.

[90] A. Levitt, J. Zhang, G. Dion, Y. Gogotsi, J. M. Razal, Adv. Funct. Mater. 2020, n/a, 2000739.

[91] A. Szuplewska, D. Kulpińska, A. Dybko, M. Chudy, A. M. Jastrzębska, A. Olszyna, Z. Brzózka, Trends Biotechnol. 2020, 38, 264.

[92] P. J. Bora, A. G. Anil, P. C. Ramamurthy, D. Q. Tan, Mater. Adv. 2020, 1, 177.

[93] S. Neampet, N. Ruecha, J. Qin, W. Wonsawat, O. Chailapakul, N. Rodthongkum, Microchim. Acta 2019, 186, 752.

[94] Y.-Z. Zhang, Y. Wang, Q. Jiang, J. K. El-Demellawi, H. Kim, H. N. Alshareef, Adv. Mater. 2020, 32, 1908486.

[95] C. (John) Zhang, L. McKeon, M. P. Kremer, S.-H. Park, O. Ronan, A. Seral-Ascaso, S. Barwich, C. Ó. Coileáin, N. McEvoy, H. C. Nerl, B. Anasori, J. N. Coleman, Y. Gogotsi, V. Nicolosi, Nat. Commun. 2019, 10, 1795.

[96] E. A. Mayerberger, O. Urbanek, R. M. McDaniel, R. M. Street, M. W. Barsoum, C. L. 
Schauer, J. Appl. Polym. Sci. 2017, 134, 45295.

[97] C. (John) Zhang, M. P. Kremer, A. Seral-Ascaso, S.-H. Park, N. McEvoy, B. Anasori, Y. Gogotsi, V. Nicolosi, Adv. Funct. Mater. 2018, 28, 1705506.

[98] S. Kumar, Y. Lei, N. H. Alshareef, M. A. Quevedo-Lopez, K. N. Salama, Biosens. Bioelectron. 2018, 121, 243.

[99] A. S. and S. W. and E. B. and J. E.-D. and Y. Z. and A. H. and V. D. and A. Y. and G. L. and H. N. A. and S. Inal, J. Phys. Mater. 2020.

[100] J. Chen, P. Tong, L. Huang, Z. Yu, D. Tang, Electrochim. Acta 2019, 319, 375.

[101] P. K. Kalambate, N. S. Gadhari, X. Li, Z. Rao, S. T. Navale, Y. Shen, V. R. Patil, Y. Huang, TrAC Trends Anal. Chem. 2019, 120, 115643.

[102] S. Wang, P. Zeng, X. Zhu, C. Lei, Y. Huang, Z. Nie, Anal. Chem. 2020, 92, 8819.

[103] H. Zhang, Z. Wang, F. Wang, Y. Zhang, H. Wang, Y. Liu, Anal. Chem. 2020, 92, 5546.

[104] A. M. Youssef, S. A. Mohamed, M. S. Abdel-Aziz, M. E. Abdel-Aziz, G. Turky, S. Kamel, Carbohydr. Polym. 2016, 147, 333.

[105] J. Stejskal, M. Trchová, P. Bober, Z. Morávková, D. Kopecký, M. Vrňata, J. Prokeš, M. Varga, E. Watzlová, RSC Adv. 2016, 6, 88382.

[106] N. Amanat, N. L. James, D. R. McKenzie, Med. Eng. Phys. 2010, 32, 690.

[107] F. J. Carrión, J. Sanes, M.-D. Bermúdez, A. Arribas, Tribol. Lett. 2011, 41, 199.

[108] D. Ju, D. Kim, H. Yook, J. W. Han, K. Cho, Adv. Funct. Mater. 2019, 29, 1905590.

[109] F. Ahmed, B. S. Lalia, V. Kochkodan, N. Hilal, R. Hashaikeh, Desalination 2016, 391, 1.

[110] S. Shrivastava, N. Jadon, R. Jain, TrAC Trends Anal. Chem. 2016, 82, 55.

[111] G. Kaur, R. Adhikari, P. Cass, M. Bown, P. Gunatillake, RSC Adv. 2015, 5, 37553.

[112] C. Gilhotra, M. Chander, Sanjay, AIP Conf. Proc. 2019, 2142, 150008.

[113] T.-H. Le, Y. Kim, H. Yoon, Electrical and Electrochemical Properties of Conducting Polymers. Polym. 2017, 9.

[114] A. M. Grancarić, I. Jerković, V. Koncar, C. Cochrane, F. M. Kelly, D. Soulat, X. Legrand, J. Ind. Text. 2017, 48, 612.

[115] S. Meer, A. Kausar, T. Iqbal, Polym. Plast. Technol. Eng. 2016, 55, 1416.

[116] Y. Wen, J. Xu, J. Polym. Sci. Part A Polym. Chem. 2017, 55, 1121.

[117] R. Cherrington, J. Liang, Goodship, V.; Middleton, B.; Cherrington, R. B. T.-D. and M. of P. C. for M., Eds.; William Andrew Publishing: Oxford, 2016; pp. 19-51.

[118] X. Wang, F. Meng, T. Wang, C. Li, H. Tang, Z. Gao, S. Li, F. Jiang, J. Xu, J. Alloys Compd. 2018, 734, 121.

[119] D. Li, W.-Y. Lai, Y.-Z. Zhang, W. Huang, Adv. Mater. 2018, 30, 1704738.

[120] D. Wang, Y. Mei, G. Huang, J. Semicond. 2018, 39, 11002.

[121] T. Kim, S. Park, J. Seo, C. W. Lee, J.-M. Kim, Org. Electron. 2019, 74, 77.

[122] N. Kim, S. Kee, S. H. Lee, B. H. Lee, Y. H. Kahng, Y.-R. Jo, B.-J. Kim, K. Lee, Adv. Mater. 2014, 26, 2268.

[123] Y.-C. Chuang, C. Chermansky, M. Kashyap, P. Tyagi, Expert Opin. Investig. Drugs 2016, 25, 521.

[124] S. H. Lee, H. Park, W. Son, H. H. Choi, J. H. Kim, J. Mater. Chem. A 2014, 2, 13380.

[125] Q. Wei, M. Mukaida, Y. Naitoh, T. Ishida, Adv. Mater. 2013, 25, 2831.

[126] H. Shi, C. Liu, Q. Jiang, J. Xu, Adv. Electron. Mater. 2015, 1, 1500017.

[127] S. Mukherjee, R. Singh, S. Gopinathan, S. Murugan, S. Gawali, B. Saha, J. Biswas, S. Lodha, A. Kumar, ACS Appl. Mater. Interfaces 2014, 6, 17792.

[128] A. Mazaheripour, S. Majumdar, D. Hanemann-Rawlings, E. M. Thomas, C. McGuiness,

L. d'Alencon, M. L. Chabinyc, R. A. Segalman, Chem. Mater. 2018, 30, 4816.

[129] B. Sarkar, M. Jaiswal, D. K. Satapathy, J. Phys. Condens. Matter 2018, 30, 225101.

[130] S. R. Dupont, F. Novoa, E. Voroshazi, R. H. Dauskardt, Adv. Funct. Mater. 2014, 24 , 
1325.

[131] S. Chen, L. Song, Z. Tao, X. Shao, Y. Huang, Q. Cui, X. Guo, Org. Electron. 2014, 15, 3654.

[132] G. R. P., S. R. V., A. Kanwat, J. Jang, Mater. Res. Bull. 2016, 74, 346.

[133] A. Elschner, Sol. Energy Mater. Sol. Cells 2011, 95, 1333.

[134] S. A. Rutledge, A. S. Helmy, ACS Appl. Mater. Interfaces 2015, 7, 3940.

[135] T. Distler, A. R. Boccaccini, Acta Biomater. 2020, 101, 1.

[136] J.-M. Moon, N. Thapliyal, K. K. Hussain, R. N. Goyal, Y.-B. Shim, Biosens. Bioelectron. 2018, 102, 540.

[137] J. Liu, M. Agarwal, K. Varahramyan, Sensors Actuators B Chem. 2008, 135, 195.

[138] M. Mazloum-Ardakani, E. Amin-Sadrabadi, A. Khoshroo, J. Electroanal. Chem. 2016, $775,116$.

[139] M. Mojtabavi, G. Jodhani, R. Rao, J. Zhang, P. Gouma, Adv. Device Mater. 2016, 2, 1.

[140] A. Ramanavičius, A. Ramanavičienè, A. Malinauskas, Electrochim. Acta 2006, 51, 6025.

[141] G. Wang, A. Morrin, M. Li, N. Liu, X. Luo, J. Mater. Chem. B 2018, 6, 4173.

[142] Y. Zhang, T. Ji, S. Hou, L. Zhang, Y. Shi, J. Zhao, X. Xu, J. Power Sources 2018, 403, 109.

[143] Z. Wang, Q. Zhang, S. Long, Y. Luo, P. Yu, Z. Tan, J. Bai, B. Qu, Y. Yang, J. Shi, H. Zhou, Z.-Y. Xiao, W. Hong, H. Bai, ACS Appl. Mater. Interfaces 2018, 10, 10437.

[144] A. T. Cullen, A. D. Price, Smart Mater. Struct. 2019, 28, 104007.

[145] C. C. Villarreal, T. Pham, P. Ramnani, A. Mulchandani, Curr. Opin. Electrochem. 2017, $3,106$.

[146] V. B. Mohan, K. Lau, D. Hui, D. Bhattacharyya, Compos. Part B Eng. 2018, 142, 200.

[147] F. Xie, M. Yang, M. Jiang, X.-J. Huang, W.-Q. Liu, P.-H. Xie, TrAC Trends Anal. Chem. 2019, 119, 115624.

[148] A. Nag, A. Mitra, S. C. Mukhopadhyay, Sensors Actuators A Phys. 2018, 270, 177.

[149] X. Bo, M. Zhou, L. Guo, Biosens. Bioelectron. 2017, 89, 167.

[150] M. S. Dresselhaus, G. Dresselhaus, R. Saito, A. Jorio, Phys. Rep. 2005, 409, 47.

[151] S. Kumar, M. Nehra, D. Kedia, N. Dilbaghi, K. Tankeshwar, K.-H. Kim, Prog. Energy Combust. Sci. 2018, 64, 219.

[152] S. K. Vashist, D. Zheng, G. Pastorin, K. Al-Rubeaan, J. H. T. Luong, F.-S. Sheu, Carbon N. Y. 2011, 49, 4077.

[153] Y. Wang, G. J. Weng, Meguid, S. A.; Weng, G. J., Eds.; Springer International Publishing: Cham, 2018; pp. 123-156.

[154] C.-M. Tîlmaciu, M. C. Morris, Carbon nanotube biosensors .Front. Chem. 2015, 3, 59.

[155] M. Sireesha, V. Jagadeesh Babu, A. S. Kranthi Kiran, S. Ramakrishna, Nanocomposites 2018, $4,36$.

[156] T. A. Silva, F. C. Moraes, B. C. Janegitz, O. Fatibello-Filho, J. Nanomater. 2017, 2017, 4571614.

[157] E. M. Sullivan, Y. J. Oh, R. A. Gerhardt, B. Wang, K. Kalaitzidou, J. Polym. Res. 2014, $21,563$.

[158] W. S. Bao, S. A. Meguid, Z. H. Zhu, M. J. Meguid, Nanotechnology 2011, 22, 485704.

[159] E. M. Sullivan, P. Karimineghlani, M. Naraghi, R. A. Gerhardt, K. Kalaitzidou, Eur. Polym. J. 2016, 77, 31.

[160] W. A. D. M. Jayathilaka, A. Chinnappan, S. Ramakrishna, J. Mater. Chem. C 2017, 5, 9209.

[161] Y. Yi, G. Weinberg, M. Prenzel, M. Greiner, S. Heumann, S. Becker, R. Schlögl, Catal. Today 2017, 295, 32. 
[162] P. M. Lee, Z. Wang, X. Liu, Z. Chen, E. Liu, Thin Solid Films 2015, 584, 85.

[163] L. Cheng, J. Liu, S. Dong, Anal. Chim. Acta 2000, 417, 133.

[164] H. Han, H. Tachikawa, Front. Biosci. 2005, 10, 931.

[165] L. Wang, Y. Wang, Q. Zhuang, J. Electroanal. Chem. 2019, 851, 113446.

[166] E. Asadian, M. Ghalkhani, S. Shahrokhian, Sensors Actuators B Chem. 2019, 293, 183.

[167] A. S. Arribas, E. Bermejo, M. Chicharro, A. Zapardiel, G. L. Luque, N. F. Ferreyra, G. A. Rivas, Anal. Chim. Acta 2007, 596, 183.

[168] C. B. Sweeney, B. A. Lackey, M. J. Pospisil, T. C. Achee, V. K. Hicks, A. G. Moran, B. R. Teipel, M. A. Saed, M. J. Green, Sci. Adv. 2017, 3, e1700262.

[169] G. V Salmoria, R. A. Paggi, A. Lago, V. E. Beal, Polym. Test. 2011, 30, 611.

[170] S. Yuan, J. Bai, C. K. Chua, J. Wei, K. Zhou, Polymers (Basel). 2016, 8, 370.

[171] Q. Mu, L. Wang, C. K. Dunn, X. Kuang, F. Duan, Z. Zhang, H. J. Qi, T. Wang, Addit. Manuf. 2017, 18, 74.

[172] Y. Zuo, X. Su, X. Li, Z. Yao, T. Yu, J. Zhou, J. Li, J. Lu, J. Ding, Carbon N. Y. 2020, $167,62$.

[173] S. W. Kwok, K. H. H. Goh, Z. D. Tan, S. T. M. Tan, W. W. Tjiu, J. Y. Soh, Z. J. G. Ng, Y. Z. Chan, H. K. Hui, K. E. J. Goh, Appl. Mater. Today 2017, 9, 167.

[174] W. Xiong, Y. Liu, L. J. Jiang, Y. S. Zhou, D. W. Li, L. Jiang, J.-F. Silvain, Y. F. Lu, Adv. Mater. 2016, 28, 2002.

[175] L. Lin, N. Ecke, M. Huang, X.-Q. Pei, A. K. Schlarb, Compos. Part B Eng. 2019, 177, 107428.

[176] W. Liu, N. Wu, K. Pochiraju, Compos. Part A Appl. Sci. Manuf. 2018, 108, 1.

[177] M. Das, V. K. Balla, In Additive Manufacturing; CRC Press, 2015; pp. 310-345.

[178] M. Mahmoudpour, J. Ezzati Nazhad Dolatabadi, M. Torbati, A. Pirpour Tazehkand, A. Homayouni-Rad, M. de la Guardia, Biosens. Bioelectron. 2019, 143, 111603.

[179] A. Manikandan, Y.-Z. Chen, C.-C. Shen, C.-W. Sher, H.-C. Kuo, Y.-L. Chueh, Prog. Quantum Electron. 2019, 68, 100226.

[180] T. C. Canevari, M. Nakamura, F. H. Cincotto, F. M. de Melo, H. E. Toma, Electrochim. Acta 2016, 209, 464.

[181] Q. Huang, X. Lin, L. Tong, Q.-X. Tong, ACS Sustain. Chem. Eng. 2020, 8, 1644.

[182] X. Ran, Q. Qu, X. Qian, W. Xie, S. Li, L. Li, L. Yang, Sensors Actuators B Chem. 2018, 257,362 .

[183] H. Mahmoudi-Moghaddam, S. Tajik, H. Beitollahi, Microchem. J. 2019, 150, 104085.

[184] M. B. Gholivand, E. Ahmadi, M. Mavaei, Sensors Actuators B Chem. 2019, 299, 126975.

[185] Y.-C. Chen, W.-H. Chiang, D. Kurniawan, P.-C. Yeh, K. Otake, C.-W. Kung, ACS Appl. Mater. Interfaces 2019, 11, 35319.

[186] C. D. Brubaker, T. M. Frecker, J. R. McBride, K. R. Reid, G. K. Jennings, S. J. Rosenthal, D. E. Adams, J. Mater. Chem. C 2018, 6, 7584.

[187] Y. Zhou, K. J. Mintz, C. Y. Oztan, S. D. Hettiarachchi, Z. Peng, E. S. Seven, P. Y. Liyanage, S. De La Torre, E. Celik, R. M. Leblanc, Polymers (Basel). 2018, 10, 921.

[188] J. Chen, Y. Wu, X. Li, F. Cao, Y. Gu, K. Liu, X. Liu, Y. Dong, J. Ji, H. Zeng, Adv. Mater. Technol. 2017, 2, 1700132.

[189] C. Zhang, K. Shen, B. Li, S. Li, S. Yang, J. Mater. Chem. A 2018, 6, 19960.

[190] K. Shen, J. Ding, S. Yang, Adv. Energy Mater. 2018, 8, 1800408.

[191] S. A. Zaidi, RSC Adv. 2016, 6, 88807.

[192] Y. Cao, T. Feng, J. Xu, C. Xue, Biosens. Bioelectron. 2019, 141, 111447.

[193] L. Abbasy, A. Mohammadzadeh, M. Hasanzadeh, N. Razmi, J. Pharm. Biomed. Anal. $2020,188,113447$.

[194] N. Li, Y. Liu, F. Liu, M. Luo, Y. Wan, Z. Huang, Q. Liao, F. Mei, Z. Wang, A. Jin, Y. 
Shi, B. Lu, Acta Biomater. 2017, 51, 175.

[195] R. Aeinehvand, P. Zahedi, S. Kashani-Rahimi, M. Fallah-Darrehchi, M. Shamsi, Polym. Adv. Technol. 2017, 28, 828.

[196] F. Canfarotta, A. Waters, R. Sadler, P. McGill, A. Guerreiro, D. Papkovsky, K. Haupt, S. Piletsky, Nano Res. 2016, 9, 3463.

[197] G. Zhu, W. Li, P. Wang, G. Cheng, L. Chen, K. Zhang, X. Li, J. Sep. Sci. 2020, 43, 639.

[198] M. Singh, S. Singh, S. P. Singh, S. S. Patel, Trends Environ. Anal. Chem. 2020, 27, e00092.

[199] A. G. Ayankojo, J. Reut, V. Ciocan, A. Öpik, V. Syritski, Talanta 2020, 209, 120502.

[200] G. F. de Oliveira, F. F. Hudari, F. M. V Pereira, M. V. B. Zanoni, J. L. da Silva, ChemElectroChem 2020, 7, 3006.

[201] R. R. Pupin, M. V. Foguel, L. M. Gonçalves, M. del P. T. Sotomayor, J. Appl. Polym. Sci. 2020, 137, 48496.

[202] J. C. Yang, J. Lee, S. W. Hong, J. Park, Sensors Actuators B Chem. 2020, 320, 128366.

[203] L. P. C. Gomez, A. Spangenberg, X.-A. Ton, Y. Fuchs, F. Bokeloh, J.-P. Malval, B. Tse Sum Bui, D. Thuau, C. Ayela, K. Haupt, O. Soppera, Adv. Mater. 2016, 28, 5931.

[204] X. Yang, J. Sun, F. Cui, J. Ji, L. Wang, Y. Zhang, X. Sun, Talanta 2020, 219, 121343.

[205] V. Thiviyanathan, D. G. Gorenstein, PROTEOMICS - Clin. Appl. 2012, 6, 563.

[206] R. Reid, B. Chatterjee, S. J. Das, S. Ghosh, T. K. Sharma, Anal. Biochem. 2020, 593, 113574.

[207] S. Xie, Y. Du, Y. Zhang, Z. Wang, D. Zhang, L. He, L. Qiu, J. Jiang, W. Tan, Nat. Commun. 2020, 11, 1347.

[208] P. Bayat, R. Nosrati, M. Alibolandi, H. Rafatpanah, K. Abnous, M. Khedri, M. Ramezani, Biochimie 2018, 154, 132.

[209] J. Zhou, J. Rossi, Nat. Rev. Drug Discov. 2017, 16, 181.

[210] P. Jolly, V. Tamboli, R. L. Harniman, P. Estrela, C. J. Allender, J. L. Bowen, Biosens. Bioelectron. 2016, 75, 188.

[211] M. Roushani, F. Shahdost-fard, Bioelectrochemistry 2019, 126, 38.

[212] H. Munawar, J. S. Mankar, M. D. Sharma, A. Garcia-Cruz, L. A. L. Fernandes, M. Peacock, R. J. Krupadam, Talanta 2020, 219, 121273.

[213] S. M. Mugo, J. Alberkant, Anal. Bioanal. Chem. 2020, 412, 1825.

[214] S. Díaz-Amaya, M. Zhao, L.-K. Lin, C. Ostos, J. P. Allebach, G. T.-C. Chiu, A. J. Deering, L. A. Stanciu, Small 2019, 15, 1805342.

[215] J. Jaeger, F. Groher, J. Stamm, D. Spiehl, J. Braun, E. Dörsam, B. Suess, Biosensors 2019, 9,7 .

[216] W. M. Rockey, L. Huang, K. C. Kloepping, N. J. Baumhover, P. H. Giangrande, M. K. Schultz, Bioorg. Med. Chem. 2011, 19, 4080.

[217] A. Chakraborty, D. N. Tibarewala, A. Barui, In Woodhead Publishing Series in Electronic and Optical Materials; Pal, K.; Kraatz, H.-B.; Khasnobish, A.; Bag, S.; Banerjee, I.; Kuruganti, U. B. T.-B. and M. D., Eds.; Woodhead Publishing, 2019; pp. 97-122.

[218] M. R. Dunn, C. M. McCloskey, P. Buckley, K. Rhea, J. C. Chaput, J. Am. Chem. Soc. 2020, 142, 7721.

[219] A. A. Shoara, O. Reinstein, O. A. Borhani, T. R. Martin, S. Slavkovic, Z. R. Churcher, P. E. Johnson, Biochimie 2018, 145, 137.

[220] A. Awad, S. J. Trenfield, S. Gaisford, A. W. Basit, Int. J. Pharm. 2018, 548, 586.

[221] S. J. Trenfield, A. Awad, A. Goyanes, S. Gaisford, A. W. Basit, Trends Pharmacol. Sci. 2018, 39, 440.

[222] M. E. E.-J. L. R.-A. E.-B. A. S. Cruz, IntechOpen: Rijeka, 2018; p. Ch. 3.

[223] T. Kuipers, W. Elkhuizen, J. Verlinden, E. Doubrovski, Comput. Graph. 2018, 74, 23. 
[224] F. Fina, A. Goyanes, M. Rowland, S. Gaisford, A. W Basit, Polymers (Basel). 2020, 12, 1769.

[225] K. K. Guduru, G. Srinivasu, Mater. Today Proc. 2020.

[226] L. Chen, X. Zhang, Y. Wang, T. A. Osswald, Polym. Compos. 2020, 41, 1356.

[227] F. Lambiase, S. Genna, C. Leone, Opt. Lasers Eng. 2020, 124, 105801.

[228] M. E. Carkaci, M. Secmen, Int. J. RF Microw. Comput. Eng. 2020, 30, e22062.

[229] C. Kalinke, N. V. Neumsteir, P. R. de Oliveira, B. C. Janegitz, J. A. Bonacin, Anal. Chim. Acta 2020.

[230] S. V. F. Castro, A. P. Lima, R. G. Rocha, R. M. Cardoso, R. H. O. Montes, M. H. P. Santana, E. M. Richter, R. A. A. Munoz, Anal. Chim. Acta 2020, 1130, 126.

[231] C. W. Foster, H. M. Elbardisy, M. P. Down, E. M. Keefe, G. C. Smith, C. E. Banks, Chem. Eng. J. 2020, 381, 122343.

[232] Z. Rymansaib, P. Iravani, E. Emslie, M. Medvidović-Kosanović, M. Sak-Bosnar, R. Verdejo, F. Marken, Electroanalysis 2016, 28, 1517.

[233] S. Bose, R. A. Khare, P. Moldenaers, Polymer (Guildf). 2010, 51, 975.

[234] K. Ahmed, M. Kawakami, A. Khosla, H. Furukawa, Polym. J. 2019, $51,511$.

[235] S. Shi, Y. Chen, J. Jing, L. Yang, RSC Adv. 2019, 9, 29980.

[236] A. Abdalla, H. H. Hamzah, O. Keattch, D. Covill, B. A. Patel, Electrochim. Acta 2020, 354, 136618.

[237] M. Spoerk, J. Gonzalez-Gutierrez, J. Sapkota, S. Schuschnigg, C. Holzer, Plast. Rubber Compos. 2018, 47, 17.

[238] N. Aliheidari, R. Tripuraneni, A. Ameli, S. Nadimpalli, Polym. Test. 2017, 60, 94.

[239] A. Adams, A. Malkoc, J. T. La Belle, J. Diabetes Sci. Technol. 2017, 12, 176.

[240] W. A. Gebreyes, L. Migliorini, F. Pezzotta, Y. Shacham-Diamand, T. Santaniello, P. Milani, Sensors Actuators A Phys. 2020, 301, 111706.

[241] V. Katseli, A. Economou, C. Kokkinos, Talanta 2020, 208, 120388.

[242] G. D. da Silveira, R. F. Quero, L. P. Bressan, J. A. Bonacin, D. P. de Jesus, J. A. F. da Silva, Anal. Chim. Acta 2020.

[243] R. G. Rocha, R. M. Cardoso, P. J. Zambiazi, S. V. F. Castro, T. V. B. Ferraz, G. de O. Aparecido, J. A. Bonacin, R. A. A. Munoz, E. M. Richter, Anal. Chim. Acta 2020, 1132, 1.

[244] T.-T. Wang, Z.-L. Huang, Q. Xu, X.-Y. Hu, Int. J. Environ. Anal. Chem. 2018, 98, 477.

[245] A. M. López Marzo, C. C. Mayorga-Martinez, M. Pumera, Biosens. Bioelectron. 2020, $151,111980$.

[246] M. P. Browne, F. Novotný, Z. Sofer, M. Pumera, ACS Appl. Mater. Interfaces 2018, 10, 40294.

[247] P. L. dos Santos, V. Katic, H. C. Loureiro, M. F. dos Santos, D. P. dos Santos, A. L. B. Formiga, J. A. Bonacin, Sensors Actuators B Chem. 2019, 281, 837.

[248] L. Lin, X. Xu, C. Chu, M. K. Majeed, J. Yang, Angew. Chemie 2016, 128, 14269.

[249] R. M. Cardoso, P. R. L. Silva, A. P. Lima, D. P. Rocha, T. C. Oliveira, T. M. do Prado, E. L. Fava, O. Fatibello-Filho, E. M. Richter, R. A. A. Muñoz, Sensors Actuators B Chem. 2020, 307, 127621.

[250] E. Vaněčková, M. Bouša, R. Sokolová, P. Moreno-García, P. Broekmann, V. Shestivska, J. Rathouský, M. Gál, T. Sebechlebská, V. Kolivoška, J. Electroanal. Chem. 2020, 858, 113763.

[251] E. Vaněčková, M. Bouša, Š. Nováková Lachmanová, J. Rathouský, M. Gál, T. Sebechlebská, V. Kolivoška, J. Electroanal. Chem. 2020, 857, 113745.

[252] K. P. A. Kumar, K. Ghosh, O. Alduhaish, M. Pumera, Electrochem. commun. 2020, 120, 106827.

[253] Y. Mou, H. Cheng, Y. Peng, M. Chen, Mater. Lett. 2018, 229, 353. 
[254] W. Nyabeze, B. McFadzean, Miner. Eng. 2016, 92, 28.

[255] G. Kollamaram, D. M. Croker, G. M. Walker, A. Goyanes, A. W. Basit, S. Gaisford, Int. J. Pharm. 2018, 545, 144.

[256] Y.-Q. Zhao, J.-H. Yang, X. Ding, X. Ding, S. Duan, F.-J. Xu, Bioact. Mater. 2020, 5, 185.

[257] V. A. O. P. da Silva, W. S. Fernandes-Junior, D. P. Rocha, J. S. Stefano, R. A. A. Munoz, J. A. Bonacin, B. C. Janegitz, Biosens. Bioelectron. 2020, 112684.

[258] M. Wei, F. Zhang, W. Wang, P. Alexandridis, C. Zhou, G. Wu, J. Power Sources 2017, 354, 134.

[259] L. Nayak, S. Mohanty, S. K. Nayak, A. Ramadoss, J. Mater. Chem. C 2019, 7, 8771.

[260] M. Elbadawi, J. Meredith, L. Hopkins, I. Reaney, Adv. Tech. Bone Regen. 2016.

[261] J. K. Nolan, T. N. H. Nguyen, K. V. H. Le, L. E. DeLong, H. Lee, SLAS Technol. Transl. Life Sci. Innov. 2019, 25, 33.

[262] T. N. H. Nguyen, J. K. Nolan, X. Cheng, H. Park, Y. Wang, S. Lam, H. Lee, S. J. Kim, R. Shi, A. A. Chubykin, H. Lee, J. Electroanal. Chem. 2020, 866, 114136.

[263] Y. Dong, X. Min, W. S. Kim, IEEE Sens. J. 2018, 18, 2959.

[264] T. N. H. Nguyen, J. K. Nolan, H. Park, S. Lam, M. Fattah, J. C. Page, H.-E. Joe, M. B. G. Jun, H. Lee, S. J. Kim, R. Shi, H. Lee, Biosens. Bioelectron. 2019, 131, 257.

[265] T. N. H. Nguyen, X. Jin, J. K. Nolan, J. Xu, K. V. H. Le, S. Lam, Y. Wang, M. A. Alam, H. Lee, ACS Biomater. Sci. Eng. 2020, 6, 5315.

[266] Y. Sui, A. Hess-Dunning, R. M. Sankaran, C. A. Zorman, J. Microelectromechanical Syst. 2020, 29, 1026.

[267] S. J. Trenfield, H. Xian Tan, A. Awad, A. Buanz, S. Gaisford, A. W. Basit, A. Goyanes, Int. J. Pharm. 2019, 567, 118443.

[268] D. A. Anderegg, H. A. Bryant, D. C. Ruffin, S. M. Skrip, J. J. Fallon, E. L. Gilmer, M. J. Bortner, Addit. Manuf. 2019, 26, 76.

[269] M. Elbadawi, T. Gustaffson, S. Gaisford, A. W. Basit, Int. J. Pharm. 2020, 590, 119868.

[270] T.-H. Kang, S.-W. Lee, K. Hwang, W. Shim, K.-Y. Lee, J.-A. Lim, W.-R. Yu, I.-S. Choi, H. Yi, ACS Appl. Mater. Interfaces 2020, 12, 24231.

[271] A. Phongphut, C. Sriprachuabwong, A. Wisitsoraat, A. Tuantranont, S. Prichanont, P. Sritongkham, Sensors Actuators B Chem. 2013, 178, 501.

[272] B. Nagar, M. Jović, V. C. Bassetto, Y. Zhu, H. Pick, P. Gómez-Romero, A. Merkoçi, H. H. Girault, A. Lesch, ChemElectroChem 2020, 7, 460.

[273] B. Weng, A. Morrin, R. Shepherd, K. Crowley, A. J. Killard, P. C. Innis, G. G. Wallace, J. Mater. Chem. B 2014, 2, 793.

[274] E. Bihar, S. Wustoni, A. M. Pappa, K. N. Salama, D. Baran, S. Inal, npj Flex. Electron. 2018, 2, 30.

[275] R. Shiwaku, H. Matsui, K. Nagamine, M. Uematsu, T. Mano, Y. Maruyama, A. Nomura, K. Tsuchiya, K. Hayasaka, Y. Takeda, T. Fukuda, D. Kumaki, S. Tokito, Sci. Rep. 2018, 8,6368 .

[276] I. J. Fernandes, A. F. Aroche, A. Schuck, P. Lamberty, C. R. Peter, W. Hasenkamp, T. L. A. C. Rocha, Sci. Rep. 2020, 10, 8878.

[277] J. Kudr, L. Zhao, E. P. Nguyen, H. Arola, T. K. Nevanen, V. Adam, O. Zitka, A. Merkoçi, Biosens. Bioelectron. 2020, 156, 112109.

[278] M. A. Ali, C. Hu, S. Jahan, B. Yuan, M. S. Saleh, E. Ju, S.-J. Gao, R. P. Panat, medRxiv 2020.

[279] S. Zips, L. Grob, P. Rinklin, K. Terkan, N. Y. Adly, L. J. K. Weiß, D. Mayer, B. Wolfrum, ACS Appl. Mater. Interfaces 2019, 11, 32778.

[280] N. G. Di Novo, E. Cantù, S. Tonello, E. Sardini, M. Serpelloni, Sensors 2019, 19, 1842. [281] E. Cantù, S. Tonello, G. Abate, D. Uberti, E. Sardini, M. Serpelloni, Sensors 2018, 18, 
3719.

[282] K. Parate, C. C. Pola, S. V Rangnekar, D. L. Mendivelso-Perez, E. A. Smith, M. C. Hersam, C. L. Gomes, J. C. Claussen, 2D Mater. 2020, 7, 34002.

[283] H. Yang, M. T. Rahman, D. Du, R. Panat, Y. Lin, Sensors Actuators B Chem. 2016, 230, 600.

[284] W. Skorupa, T. Schumann, L. Rebohle, Surf. Coatings Technol. 2017, 314, 169.

[285] J. Schnitker, N. Adly, S. Seyock, B. Bachmann, A. Yakushenko, B. Wolfrum, A. Offenhäusser, Adv. Biosyst. 2018, 2, 1700136.

[286] X. Xu, A. Awad, P. R. Martinez, S. Gaisford, A. Goyanes, A. W. Basit, J. Control. Release 2020.

[287] M. Revilla-León, M. Özcan, J. Prosthodont. 2019, 28, 146.

[288] G. Scordo, V. Bertana, L. Scaltrito, S. Ferrero, M. Cocuzza, S. L. Marasso, S. Romano, R. Sesana, F. Catania, C. F. Pirri, Mater. Today Commun. 2019, 19, 12.

[289] G. Gonzalez, A. Chiappone, I. Roppolo, E. Fantino, V. Bertana, F. Perrucci, L. Scaltrito, F. Pirri, M. Sangermano, Polymer (Guildf). 2017, 109, 246.

[290] B. Rezaei, J. Y. Pan, C. Gundlach, S. S. Keller, Mater. Des. 2020, 193, 108834.

[291] B. Chen, B. Zhang, C. Chen, J. Hu, J. Qi, T. He, P. Tian, X. Zhang, G. Ni, M. M.-C. Cheng, Biomed. Microdevices 2020, 22, 43.

[292] M. A. Haque, N. V Lavrik, A. Hedayatipour, D. Hensley, D. P. Briggs, N. McFarlane, J. Vac. Sci. Technol. B 2020, 38, 52203.

[293] C. Yang, Q. Cao, P. Puthongkham, S. T. Lee, M. Ganesana, N. V Lavrik, B. J. Venton, Angew. Chemie Int. Ed. 2018, 57, 14255.

[294] P. Miller, M. Moorman, R. Manginell, C. Ashlee, I. Brener, D. Wheeler, R. Narayan, R. Polsky, Electroanalysis 2016, 28, 1305.

[295] M. A. Haque, N. V Lavrik, D. Hensley, N. McFarlane, In 2019 IEEE 69th Electronic Components and Technology Conference (ECTC); 2019; pp. 2073-2078.

[296] S. S. Leong, Y. W. Yee, W. F. Edith, T. B. Yen, Z. Ziqiang, Z. Lin, T. Zhiling, Y. Shoufeng, Rapid Prototyp. J. 2017, 23, 611.

[297] S. Singh, V. S. Sharma, A. Sachdeva, Mater. Sci. Technol. 2016, 32, 760.

[298] P. Parandoush, D. Lin, Compos. Struct. 2017, 182, 36.

[299] A. Awad, F. Fina, A. Goyanes, S. Gaisford, A. W. Basit, Int. J. Pharm. 2020, 586, 119594.

[300] L.-C. Zhang, H. Attar, Adv. Eng. Mater. 2016, 18, 463.

[301] N. K. Roy, D. Behera, O. G. Dibua, C. S. Foong, M. A. Cullinan, Microsystems Nanoeng. 2019, 5, 64.

[302] A. Gisario, M. Kazarian, F. Martina, M. Mehrpouya, J. Manuf. Syst. 2019, 53, 124.

[303] A. A. Serkov, H. V Snelling, S. Heusing, T. M. Amaral, Sci. Rep. 2019, 9, 1773.

[304] F. Zacharatos, I. Theodorakos, P. Karvounis, S. Tuohy, N. Braz, S. Melamed, A. Kabla, F. la Vega, K. Andritsos, A. Hatziapostolou, D. Karnakis, I. Zergioti, Materials (Basel). 2018, 11 .

[305] J. Kwon, H. Cho, H. Eom, H. Lee, Y. D. Suh, H. Moon, J. Shin, S. Hong, S. H. Ko, ACS Appl. Mater. Interfaces 2016, 8, 11575.

[306] A. Ambrosi, J. G. S. Moo, M. Pumera, Adv. Funct. Mater. 2016, 26, 698.

[307] B. R. Liyarita, A. Ambrosi, M. Pumera, Electroanalysis 2018, 30, 1319.

[308] C. Tan, M. Z. M. Nasir, A. Ambrosi, M. Pumera, Anal. Chem. 2017, 89, 8995.

[309] Y. C. Li, E. L. Melenbrink, G. J. Cordonier, C. Boggs, A. Khan, M. K. Isaac, L. K. Nkhonjera, D. Bahati, S. J. Billinge, S. M. Haile, R. A. Kreuter, R. M. Crable, T. E. Mallouk, J. Chem. Educ. 2018, 95, 1658.

[310] S. Damiati, S. Küpcü, M. Peacock, C. Eilenberger, M. Zamzami, I. Qadri, H. Choudhry, U. B. Sleytr, B. Schuster, Biosens. Bioelectron. 2017, 94, 500. 
[311] S. Damiati, M. Peacock, S. Leonhardt, L. Damiati, M. A. Baghdadi, H. Becker, R. Kodzius, B. Schuster, Genes (Basel). 2018, 9, 89.

[312] S. C. Silva, R. M. Cardoso, E. M. Richter, R. A. A. Munoz, E. Nossol, Mater. Chem. Phys. 2020, 250, 123011.

[313] N. C. de Moraes, E. N. T. da Silva, J. M. Petroni, V. S. Ferreira, B. G. Lucca, Electrophoresis 2020, 41, 278.

[314] S. Mavrikou, G. Moschopoulou, V. Tsekouras, S. Kintzios, Sensors 2020, 20, 3121.

[315] S. A. N. Gowers, V. F. Curto, C. A. Seneci, C. Wang, S. Anastasova, P. Vadgama, G.Z. Yang, M. G. Boutelle, Anal. Chem. 2015, 87, 7763.

[316] M. Senel, M. Dervisevic, S. Alhassen, A. Alachkar, N. H. Voelcker, Anal. Chem. 2020, 92, 7746.

[317] Y. Hou, W. Tang, W. Qi, X. Guo, J. Lin, Biosens. Bioelectron. 2020, 157, 112160.

[318] G. W. Bishop, J. E. Satterwhite, S. Bhakta, K. Kadimisetty, K. M. Gillette, E. Chen, J. F. Rusling, Anal. Chem. 2015, 87, 5437.

[319] L. Krejcova, L. Nejdl, M. A. M. Rodrigo, M. Zurek, M. Matousek, D. Hynek, O. Zitka, P. Kopel, V. Adam, R. Kizek, Biosens. Bioelectron. 2014, 54, 421.

[320] F. T. C. Moreira, M. G. F. Sale, M. Di Lorenzo, Biosens. Bioelectron. 2017, 87, 607.

[321] B. Schmidt, D. King, J. Kariuki, J. Chem. Educ. 2018, 95, 2076.

[322] Y. Zhao, B. Wang, H. Hojaiji, Z. Wang, S. Lin, C. Yeung, H. Lin, P. Nguyen, K. Chiu, K. Salahi, X. Cheng, J. Tan, B. A. Cerrillos, S. Emaminejad, Sci. Adv. 2020, 6, eaaz0007.

[323] T. Glennon, C. O’Quigley, M. McCaul, G. Matzeu, S. Beirne, G. G. Wallace, F. Stroiescu, N. O’Mahoney, P. White, D. Diamond, Electroanalysis 2016, $28,1283$.

[324] A. J. Bandodkar, S. Imani, R. Nuñez-Flores, R. Kumar, C. Wang, A. M. V. Mohan, J. Wang, P. P. Mercier, Biosens. Bioelectron. 2018, 101, 181.

[325] L. Cevenini, M. M. Calabretta, G. Tarantino, E. Michelini, A. Roda, Sensors Actuators B Chem. 2016, 225, 249.

[326] I. C. Samper, S. A. N. Gowers, M. A. Booth, C. Wang, T. Watts, T. Phairatana, N. Vallant, B. Sandhu, V. Papalois, M. G. Boutelle, Anal. Chem. 2019, 91, 14631.

[327] L. P. Bressan, T. M. Lima, G. D. da Silveira, J. A. F. da Silva, SN Appl. Sci. 2020, 2, 984.

[328] Y.-T. Kwon, Y.-S. Kim, S. Kwon, M. Mahmood, H.-R. Lim, S.-W. Park, S.-O. Kang, J. J. Choi, R. Herbert, Y. C. Jang, Y.-H. Choa, W.-H. Yeo, Nat. Commun. 2020, 11, 3450.

[329] M. Saari, B. Cox, E. Richer, P. S. Krueger, A. L. Cohen, 3D Print. Addit. Manuf. 2015, 2,32 .

[330] X. Wang, M. Jiang, Z. Zhou, J. Gou, D. Hui, Compos. Part B Eng. 2017, 110, 442.

[331] A. Rahim, Z. U. Rehman, S. Mir, N. Muhammad, F. Rehman, M. H. Nawaz, M. Yaqub, S. A. Siddiqi, A. A. Chaudhry, J. Mol. Liq. 2017, 248, 425.

[332] C. D. Blanchette, J. M. Knipe, J. K. Stolaroff, J. R. DeOtte, J. S. Oakdale, A. Maiti, J. M. Lenhardt, S. Sirajuddin, A. C. Rosenzweig, S. E. Baker, Nat. Commun. 2016, 7 , 11900 . 